\title{
Analysis of sex-specific lipid metabolism in $P$. falciparum gametocytes points to importance of sphingomyelin for gametocytogenesis
}

\author{
Melanie C. Ridgway ${ }^{1}$, Daniela Cihalova ${ }^{1}$, Simon H.J. Brown ${ }^{2}$, Phuong Tran ${ }^{1}$, Todd
} W. Mitchell ${ }^{3}$, Alexander G. Maier ${ }^{1}$

${ }^{1}$ Research School of Biology, Australian National University, Canberra, Australian Capital Territory 2601, Australia

${ }^{2}$ Molecular Horizons and School of Chemistry and Molecular Biology, University of Wollongong, Wollongong, New South Wales 2522, Australia

${ }^{3}$ Illawarra Health and Medical Research Institute and School of Medicine, University of Wollongong, Wollongong, New South Wales 2522, Australia

\begin{abstract}
$\underline{\text { Abstract }}$
Male and female Plasmodium falciparum gametocytes are the parasite lifecycle stage responsible for transmission of malaria from the human host to mosquito vector. Not only are gametocytes able to survive in radically different host environments, but they are also precursors for male and female gametes that reproduce sexually soon after ingestion by the mosquito. Here we investigate the sex-specific lipid metabolism of gametocytes within their host red blood cell and poised for ingestion by the mosquito vector and subsequent sexual reproduction.

Comparison of the male and female lipidome identifies cholesteryl esters and dihydrosphingomyelin enrichment in female gametocytes. Chemical inhibition of each of these lipid types in mature gametocytes suggests dihydrosphingomyelin synthesis but not cholesteryl ester synthesis is important for sex-specific gametocyte viability. Genetic disruption of each of the two sphingomyelin synthase gene points towards sphingomyelin synthesis contributing to gametocytogenesis.

This study shows that gametocytes are not only distinct from asexual stages, but that the lipid composition is also vastly different between male and female gametocytes, reflecting the different cellular roles these stages play. Together our results highlight the sex-specific nature of gametocyte lipid metabolism that has the potential to be targeted to block malaria transmission.
\end{abstract}




\section{Introduction}

Transmission of Plasmodium falciparum from the human to mosquito host depends on male and female gametocytes. The development of transmissible gametocytes (gametocytogenesis) is by far the longest developmental sexual stage in $P$. falciparum. After 10-12 days of maturation in the human red blood cell (RBC), ingestion by a mosquito rapidly activates the gametocytes to form mature gametes capable of sexual reproduction (Bennink et al. 2016). Within 15 minutes of the blood meal, gametocytes egresses from their host RBC. While male gametocytes multiply into eight flagellated microgametes, female gametocytes form an immobile spherical macrogamete, poised for fertilisation. The zygote develops in the extracellular environment until invasion of the mosquito midgut wall 19-36 hours post blood meal (Aikawa et al. 1984; Sinden et al. 1985; Vlachou et al. 2004).

Such a rapid sex-specific metamorphosis is necessarily preceded by sex-specific preparations in the human host during the relatively slow gametocytogenesis. Surprisingly though, morphological gametocyte sex dimorphism is relatively subtle and mostly limited to the ultrastructural level. Recently the tagging of sex-specific molecular markers has revealed sex-specific gene and protein expression profiles (Lasonder et al. 2016; Miao et al. 2017). These studies identified differences in the expression of mRNA and protein involved in lipid metabolic pathways including phosphatidylcholine biosynthesis, fatty acid biosynthesis and ether lipid metabolism (Lasonder et al. 2016). Whether this results in differences in the lipid composition between male and female gametocytes remains unknown.

Lipids fulfil a vast range of functions in the cell. They not only provide building blocks in the form of fatty acids for the parasite's metabolism, but they also form the basis for membranes (mainly phospholipids and cholesterol), functionalise subdomains within membranes (e.g. sphingolipids), serve as energy storage (e.g. neutral lipids like cholesterylesters and triacylglycerol) and act as signalling molecules (e.g. diacylglycerol and ceramide). The nature of lipids in male and female gametocytes may reveal diverging cellular functions that precede sex-specific development in the mosquito. 
69 Based on their resilience against many drugs that are active against asexual forms, 70 gametocytes were generally thought to be relatively metabolically inactive (Canning 71 \& Sinden 1975; Sinden et al. 1978). However, the lipidome of combined male and 72 female gametocytes changes significantly between young and mature gametocytes 73 (Gulati et al. 2015; Tran et al. 2016), suggesting that lipid metabolism is active during 74 gametocytogenesis. In particular, Gulati et al. (2015) identified intermediate products 75 of sphingolipid metabolism in mature gametocytes, revealing that de novo 76 sphingomyelin synthesis occurs during gametocytogenesis.

78 Sphingomyelin is synthesised de novo from serine and palmitoyl-CoA in five 79 consecutive reactions. The final step of de novo sphingomyelin synthesis is mediated 80 by sphingomyelin synthase (SMS). P. falciparum encodes two SMS (Gardner et al. 81 2002), one of which is expressed throughout blood stages (SMS1, PF3D7_0625000), 82 the other of which is gametocyte specific and female enriched (SMS2, 83 PF3D7_0625100) (López-Barragán et al. 2011; Lasonder et al. 2016). In addition to 84 generating sphingomyelin, SMS also regulates the levels of secondary messenger 85 molecules such as ceramide and diacylglycerol (Fyrst \& Saba 2010). The functional 86 significance of SMS1 and SMS2 in P. falciparum has yet to be investigated.

87

88 Here we explore the sex-specific lipidome of mature P. falciparum gametocytes 89 within their host RBC to determine the respective contribution of male and female 90 gametocytes to the lipid profile of the infected RBC (iRBC). The functional 91 significance of de novo synthesis of key sex-specific lipids is then investigated by 92 pharmacological inhibition and reverse genetics. 


\section{Materials and Methods}

\section{Culturing techniques}

Asexual 3D7 strain $P$. falciparum parasites were maintained in complete culture medium (RPMI 1640-Hepes with Glutamax media supplemented with $10 \mathrm{mM}$ Dglucose, $480 \mu \mathrm{M}$ hypoxanthine, $20 \mu \mathrm{g} / \mathrm{mL}$ gentamicin, $0.375 \%$ (w/v) Albumax II and $2.5 \%$ v/v heat-inactivated human serum) as previously described (Maier \& Rug 2013). Cultures were synchronised by sorbitol treatment as described previously (Lambros \& Vanderberg 1979) and isolated from their host cells by saponin treatment (Dourmashkin et al. 1962).

Parasites were induced to form gametocytes as described by Fivelman et al. (2007) with modifications to reduce asexual parasite proliferation (Ridgway et al. 2020). Male and female gametocytes were collected by fluorescence activated cell sorting as described by Ridgway et al. (2021). Briefly, magnet enriched 3D7 gametocytes with a female-specific GFP tag (gABCG2-GFP, (Tran et al. 2014)) were stained with Hoechst 33342 and collected by fluorescence activated cell sorting on day 9 post commitment (mostly at stage IV). Hoechst staining identified gametocyte-infected from uninfected RBC, while the female-specific GFP signal distinguished male and female gametocytes.

Gametocytemia was measured every second day from day 2 post commitment by flow cytometry of an aliquot of culture stained with $50 \mu \mathrm{g} / \mathrm{mL}$ Hoechst 33342 in PBS (10.6 $\mathrm{mM} \mathrm{KH}{ }_{2} \mathrm{PO} 4,1.55 \mathrm{M} \mathrm{NaCl}, 29.7 \mathrm{mM} \mathrm{Na}_{2} \mathrm{HPO} 4, \mathrm{pH}$ 7.4) for $15 \mathrm{~min}$ at $37^{\circ} \mathrm{C}$, rinsed twice in PBS with 2,000 $\times g, 1 \mathrm{~min}$ spins and resuspended in PBS. Samples were analysed on a LSR II Flow Cytometer (BD Biosciences) detecting Hoechst 33342 in the Pacific Blue channel (350 nm excitation/461 nm emission). Parasitemia was calculated as the proportion of Hoechst 33342 positive cells in 200,000 whole, single cells as gated in FACS Diva software. Statistical significance and graphing of results was performed in GraphPad Prism 7.

\section{Sex-specific gametocyte viability assay}

Magnet enriched gametocytes were exposed to $10 \mu \mathrm{M}$ of each test compound, 200 $\mu \mathrm{M}$ artemisinin (0\% viable control) or $0.1 \%(\mathrm{v} / \mathrm{v})$ DMSO (100\% viable control) for 
$12796 \mathrm{~h}$ from day 2 to 6 post commitment in complete culture medium with $50 \mathrm{mM} \mathrm{N}$ -

128 acetyl D-glucosamine in a 96 well plate at $37^{\circ} \mathrm{C}$ in hypoxic conditions $\left(1 \% \mathrm{O}_{2}, 5 \%\right.$

$\left.129 \mathrm{CO}_{2}, 94 \% \mathrm{~N}_{2}\right)$.

130

131 Parasites were then stained in $50 \mu \mathrm{g} / \mathrm{mL}$ Hoechst 33342 and $500 \mathrm{nM}$ MitoTracker

132 Deep Red in complete culture medium supplemented with $50 \mathrm{mM} \mathrm{N}$-acetyl D-

133 glucosamine for $30 \mathrm{~min}$ at $37{ }^{\circ} \mathrm{C}$ in hypoxic conditions $\left(1 \% \mathrm{O}_{2}, 5 \% \mathrm{CO}_{2}, 94 \% \mathrm{~N}_{2}\right)$.

134 Stained cells were rinsed twice in PBS with $1,000 \times g, 5$ min spins and resuspended in

135 PBS. Single colour controls for flow cytometry consisted of i) asexual 3D7 WT

136 culture stained with $500 \mathrm{nM}$ MitoTracker Deep Red or $50 \mu \mathrm{g} / \mathrm{mL}$ Hoechst 33342 for

$13730 \mathrm{~min}$ in complete culture medium, rinsed twice in PBS with $1,000 \times g, 1 \mathrm{~min}$ spins

138 and resuspended in PBS, ii) unstained 3D7 gABCG2-GFP gametocytes suspended in

139 PBS and iii) unstained asexual 3D7 WT parasites in PBS (for the unstained control).

140 Samples were analysed on a LSR II Flow Cytometer (BD Biosciences) detecting

141 Hoechst 33342 in the Pacific Blue channel (405 nm excitation/461 nm emission),

142 MitoTracker Deep Red in the APC-Cy7 channel (644 nm excitation/665 nm emission)

143 and GFP in the FITC channel (488 $\mathrm{nm}$ excitation/509 $\mathrm{nm}$ emission). A total of

144500,000 events were recorded in each single colour control. In each sample data was

145 recorded until 500,000 female gametocytes were counted.

146

147 After applying the gating strategy to isolate male and female gametocytes (Ridgway 148 et al. 2020) the mean fluorescence intensity of MitoTracker Deep Red detected on the

149 APC-Cy7 channel was recorded for each of the gametocyte populations. Data was 150 analysed in FlowJo and statistical significance was determined by ANOVA (analysis 151 of variance) in GraphPad Prism. Cell viability is expressed as a percentage of the 152 mean fluorescence intensity of MitoTracker Deep Red in gametocytes treated with $1530.1 \%$ DMSO (100\% viable) and $200 \mu \mathrm{M}$ artemisinin treated gametocytes (0\% viable).

$154 \quad$ Generation of transgenic $P$. falciparum cell lines

155 Annotated sequence of $P$. falciparum 3D7 reference strain was sourced from the 156 Plasmodium Genomics Resource PlasmoDB (www.plasmodb.org). To disrupt SMS1 157 (PF3D7_0625000) by double recombination, a 5' fragment and a 3' fragment of 158 SMS1 locus were amplified using primers al398/al399 and al400/al401 159 (Supplementary Table 1) and cloned into a pCC-1 vector containing a human 
160 dihydrofolate reductase (hDHFR) and 5-fluorocytosine drug selection cassette (Maier

161 et al. 2008) at SacII/SpeI and EcoRI/AvrII sites to generate pCC-1/SMS1 construct

162 (Supplementary Figure 1). SMS2 (PF3D7_0625100) was also disrupted by double 163 recombination by amplifying a 5' fragment with al406/al407 primers and a 3'

164 fragment with al408/409 primers (Supplementary Table 1), which were cloned into a $165 \mathrm{pCC}-1$ vector at SacII/SpeI and EcoRI/AvrII sites to generate pCC-1/SMS2 construct 166 (Supplementary Figure 2). Given that SMS1 and SMS2 are adjacent genes, the pCC-

167 1/SMS1 and 2 construct aiming to disrupt both SMS genes was generated by cloning 168 the 5' fragment of SMS1 (amplified with al398/al399 primers) and the 3' fragment of 169 SMS2 (amplified with al408/al409 primers) at SacII/SpeI and EcoRI/AvrII sites. 170 Inserted DNA regions were confirmed by analytical restriction enzyme digest and 171 sequenced to verify constructs. Plasmids were purified using Invitrogen Purelink 172 Maxiprep kit prior to transfection.

173

$174 P$. falciparum 3D7 reference strain parasites were transfected as described previously 175 (Rug \& Maier 2013). Briefly, $400 \mu \mathrm{L}$ of $100 \mu \mathrm{g}$ of plasmid DNA in Cytomix (120 $176 \mathrm{mM} \mathrm{KCl}, 0.15 \mathrm{mM} \mathrm{CaCl}_{2}, 10 \mathrm{mM} \mathrm{K} \mathrm{HPO}_{4} / \mathrm{KH}_{2} \mathrm{PO}_{4}, 25 \mathrm{mM}$ HEPES, $2 \mathrm{mM}$ ethylene 177 glycol-bis( $\beta$-aminoethyl ether)-tetraacetic acid, $5 \mathrm{mM} \mathrm{MgCl}_{2}, \mathrm{pH}$ 7.6) was 178 electroporated into a sorbitol synchronised ring stage culture at 5\% parasitemia by $179310 \mathrm{~V}, 950 \mu \mathrm{F}$ pulse delivered in a $0.2 \mathrm{~cm}$ electrode gap cuvette placed in a BioRad 180 Genepulser II with $\infty$ capacitor. Transformed cells were immediately resuspended in 181 complete culture medium with $3 \%$ haematocrit uninfected RBC and incubated at $18237^{\circ} \mathrm{C}$ in hypoxic conditions $\left(1 \% \mathrm{O}_{2}, 5 \% \mathrm{CO}_{2}, 94 \% \mathrm{~N}_{2}\right)$. Culture medium was 183 supplemented with $2 \mathrm{nM}$ WR99210 from $4 \mathrm{~h}$ post transfection and was replaced daily 184 for 5 days post transfection, then three times a week until parasites were observed by 185 Giemsa-stained thin smears of the culture. Parasites having integrated the plasmid 186 were enriched by three 21-day off/on 2 nM WR99210 cycles then negative selection 187 was applied by adding $231 \mathrm{nM} 5$-fluorocytosine. The transgenic parasites were cloned 188 by limited dilution (Rug \& Maier 2013).

\section{DNA extraction}

190 Genomic DNA was extracted from saponin-isolated trophozoite stage parasites using 191 DNeasy Blood and Tissue kit (Qiagen) as per the manufacturer's instructions. For 192 Southern blot analysis, genomic DNA was eluted twice in $200 \mu \mathrm{L}$ elution buffer each 
193 then concentrated by adding $40 \mu \mathrm{L} 3 \mathrm{M}$ sodium acetate and $880 \mu \mathrm{L} 100 \%$ ethanol at

$1944^{\circ} \mathrm{C}$ overnight. Samples were centrifuged at $17,000 \times g$ for $30 \mathrm{~min}$ at $4^{\circ} \mathrm{C}$ then DNA 195 was washed twice in $70 \%$ ethanol with $17,000 \times g, 20$ min spins. DNA was air dried 196 then re-suspended in TE buffer (10 mM Tris base, $1 \mathrm{mM}$ EDTA, pH 8.0). DNA 197 concentration and purity was measured by NanoDrop spectrophotometer 198 (Thermofisher).

\section{Quantitative PCR}

200 The abundance of WT and knock out (KO) genotypes in co-cultures was monitored 201 by quantitative PCR (qPCR) of regions of DNA specific to each genome. SMS1 KO 202 and SMS2 KO parasites were each quantified by primers specific to the sequence of 203 the inserted hDHFR drug resistance cassette. WT parasites were distinguished from 204 SMS1 KO parasites by primers specific to the region of SMS1 disrupted by 205 homologous recombination in the SMS1 KO parasites. WT parasites in the SMS2 206 KO/WT co-cultures were similarly quantified by primers specific to the disrupted 207 section of SMS2 in the SMS2 KO parasites. The primers for this experiment are 208 described in Supplementary Table 2.

209

210 The qPCR were performed using a Light Cycler 480 SYBR Green I Master mix 211 (Roche) as per the manufacturer's instructions for $10 \mu \mathrm{L}$ reactions in a 384 well plate. 212 Thermocycling was performed with a $10 \mathrm{~min}, 95^{\circ} \mathrm{C}$ pre-incubation; 45 cycles of $15 \mathrm{~s}$ 213 denaturation at $95^{\circ} \mathrm{C}, 15 \mathrm{~s}$ annealing at $52^{\circ} \mathrm{C}$ and $20 \mathrm{~s}$ elongation at $72^{\circ} \mathrm{C}$; followed by 214 a melt curve established by denaturing at $95^{\circ} \mathrm{C}$ for $30 \mathrm{~s}$, annealing at $60^{\circ} \mathrm{C}$ for $30 \mathrm{~s}$ 215 and then slowly denaturing by increasing the temperature to $95^{\circ} \mathrm{C}$ at $0.11^{\circ} \mathrm{C} / \mathrm{s}$. Melt 216 curves were observed using Light Cycler 480 software. The exported text file was 217 converted using Light Cycler 480 converter (Roche) and Cq and PCR efficiencies 218 were determined using the LinReg program (Ruijter et al. 2009). Relative 219 quantification of transcripts was expressed as described previously (Pfaffl 2001).

220

221 For the quantification of WT and KO parasite genotypes in co-cultures:

222 ratio $=\left(\mathrm{E}_{\text {target }} \Delta\right.$ CPtarget $($ clonal-competition $\left.)\right) /\left(\mathrm{E}_{\text {ref }}^{\Delta \mathrm{CPref}(\text { clonal-competition })}\right)$

223 with: $\mathrm{E}_{\text {target}}$ : primer efficiency of the target gene

$224 \quad \mathrm{E}_{\text {ref: }}$ primer efficiency of the reference gene 
255 Following transfer, the membrane was rinsed in $2 \times \mathrm{SCC}$ for 5 min then DNA was 256
$\Delta \mathrm{CP}_{\text {target }}$ (clonal-competition): difference in crossing points of target gene amplification in a clonal population and the fitness competition co-culture $\Delta \mathrm{CP}_{\text {ref }}$ (clonal-competition): difference in crossing points of reference gene amplification in a clonal population and the fitness competition co-culture

\section{Southern Blot}

Disruption of a gene locus by homologous recombination was confirmed by diagnostic digest with restriction enzymes followed by Southern blot probing for both the 5' and 3' homologous regions (Rug \& Maier 2013). DNA was digested with either AflII and PacI (for SMS1 KO screening) or Xmn I and Hind III- HF (for SMS2 KO screening) as per the manufacturer's instructions then run on a $0.8 \%$ agarose gel at 90 $\mathrm{V}$ for $15 \mathrm{~min}$ followed by a run at $20 \mathrm{~V}$ for $18 \mathrm{~h}$. The gel was then rinsed as follows: $15 \mathrm{~min}$ in depurination solution $(0.25 \mathrm{M} \mathrm{HCl})$; $3 \mathrm{~min}$ in milliQ water; $2 \times 15 \mathrm{~min}$ in denaturation solution $(0.5 \mathrm{M} \mathrm{NaOH}, 1.5 \mathrm{M} \mathrm{NaCl}) ; 3$ min in milliQ water; $2 \times 15 \mathrm{~min}$ in neutralisation solution (0.5 M Tris base, $1.5 \mathrm{M} \mathrm{NaCl}, \mathrm{pH} 7.5) ; 3 \mathrm{~min}$ in milliQ water then $5 \mathrm{~min}$ in $20 \times \mathrm{SCC}(3 \mathrm{M} \mathrm{NaCl}, 0.3 \mathrm{M}$ citric acid trisodium dihydrate, $\mathrm{pH}$ 7.0). DNA was transferred from the gel to the membrane overnight in a capillary transfer setup.

Alkali-labile digoxigenin labelled deoxyuridine triphosphate (DIG-dUTP) was incorporated in Southern blot probes by PCR using PCR DIG Probe Synthesis kit (Roche) with the following specifications (refer to sequences in Supplementary table 3). Each probe was amplified from the corresponding plasmid using either OneTaq (NEB) or Taq polymerase (Roche). SMS1 probes were purified by gel extraction (Qiagen kit). All probes were synthesised in the presence of $12 \mu \mathrm{M}$ DIG-dUTPs except the SMS2 3' probe synthesised from $6 \mu \mathrm{M}$ DIG-dUTPs. Thermocycling conditions consisted of initial denaturation for $30 \mathrm{~s}$ at $94^{\circ} \mathrm{C} ; 30$ cycles of $30 \mathrm{~s}$ at $94^{\circ} \mathrm{C}$, 1 min at $45^{\circ} \mathrm{C}\left(\mathrm{SMS} 23^{\prime}\right)$ or $50^{\circ} \mathrm{C}\left(\mathrm{SMS} 25^{\prime}\right)$ or $52^{\circ} \mathrm{C}\left(\mathrm{SMS} 15^{\prime}\right)$ or $55^{\circ} \mathrm{C}\left(\mathrm{SMS} 13^{\prime}\right)$, $1 \mathrm{~min}$ at $68^{\circ} \mathrm{C}$; final elongation for $7 \mathrm{~min}$ at $68^{\circ} \mathrm{C}$. crosslinked to the membrane in a UV cross linker set to deliver $70 \mathrm{~mJ} / \mathrm{cm}^{2}$. The membrane was incubated with gentle rocking in DIG Easy Hyb solution (Roche Cat. 
258 No. 11796895001$)$ at the respective probe hybridisation temperature $\left(\mathrm{T}_{\text {hyb }}\right)$ for 30 $259 \mathrm{~min}$. The probe was denatured in $50 \mu \mathrm{L}$ water at $95^{\circ} \mathrm{C}$ for $5 \mathrm{~min}$, cooled quickly on 260 ice then added to DIG Easy Hyb solution at $0.15 \%(\mathrm{v} / \mathrm{v})$ to prepare the hybridisation

261 solution. The membrane was incubated overnight in hybridisation solution at $\mathrm{T}_{\text {hyb }}$ with 262 gentle rocking.

263

264 The membrane was prepared for detection by rinsing in $2 \times \mathrm{SCC}$ for $5 \mathrm{~min}$; 265 equilibrating in washing buffer for $1 \mathrm{~min}(0.1 \mathrm{M}$ maleic acid, $0.15 \mathrm{M} \mathrm{NaCl}, \mathrm{pH} 7.5$ 266 with $0.3 \%(\mathrm{v} / \mathrm{v})$ Tween-20); incubating in $1 \%$ blocking solution (0.1 M maleic acid, $2670.15 \mathrm{M} \mathrm{NaCl}, \mathrm{pH} 7.5$ with $1 \%$ (w/v) skim milk powder) for $30 \mathrm{~min}$; incubating in $1 \%$ 268 blocking solution with 1:10,000 $\alpha$-Digoxigenin-AP Fab fragments (Roche) for 30 269 min; washing membrane in washing buffer for $2 \times 15 \mathrm{~min}$ then equilibrating 270 membrane in detection buffer (100 mM Tris-hydrochloride, $100 \mathrm{mM} \mathrm{NaCl}, \mathrm{pH} 9.5)$ 271 for $2 \mathrm{~min}$. The membrane was incubated in the dark with CSPD (Roche) diluted 1:100 272 in detection buffer for $5 \mathrm{~min}$ at room temperature then $10 \mathrm{~min}$ at $37^{\circ} \mathrm{C}$. The membrane 273 was exposed to Fuji Super RX-N Medical X-Ray film in an Amersham pharmacia 274 biotech hypercassette and developed film in an AGFA CP1000 photo developer.

Uninfected human RBC from six donors were pooled in three independent biological replicates each from two donors and incubated at $4 \%$ haematocrit in complete culture medium for at least $48 \mathrm{~h}$ at $37^{\circ} \mathrm{C}$ in hypoxic conditions $\left(1 \% \mathrm{O}_{2}, 5 \% \mathrm{CO}_{2}, 94 \% \mathrm{~N}_{2}\right)$.

280 Cells were pelleted at $524 \times g$ over $5 \mathrm{~min}$ and counted on an Improved Neubauer 281 haemocytometer (Hirschmann). Aliquots of $10^{7}$ cells were resuspended in $300 \mu \mathrm{L}$ of 282 methanol in $2 \mathrm{~mL}$ tough tubes (Geneworks) and stored at $-80^{\circ} \mathrm{C}$. For each biological replicate parasites of different stages where grown in the same donor batch of RBC to reduce host specific influences.

285 For collection of asexual parasite infected RBC, three independent asexual $P$. 286 falciparum cultures were sorbitol synchronised then magnet purified to more than $28798 \%$ iRBC, as determined by Giemsa-stained thin smear, and counted on an Improved 288 Neubauer haemocytometer (Hirschmann). Aliquots of $10^{7} \mathrm{iRBC}$ at $26-42 \mathrm{~h}$ post 289 invasion were resuspended in $300 \mu \mathrm{L}$ of methanol in $2 \mathrm{~mL}$ tough tubes (Geneworks) 290 and stored at $-80^{\circ} \mathrm{C}$. 
291 Male and female gametocytes were sorted live by FACS as described above, pelleted

292 at $754 \times g$ for $10 \mathrm{~min}$ and resuspended in $300 \mu \mathrm{L}$ of methanol per $10^{7}$ cells (as counted

293 during FACS) in $2 \mathrm{~mL}$ tough tubes (Geneworks). Each biological replicate of $10^{7}$

294 cells is pooled from 2-5 independent gametocyte cultures.

295

296 Lipids were extracted as described previously (Matyash et al. 2008) with 297 modifications. Internal standards (Tran et al. 2016) in $50 \mu \mathrm{L}$ of methanol with $0.01 \%$ 298 butylated hydroxytoluene were aliquoted among tubes. Internal standards and $1.4 \mathrm{~mm}$ 299 beads (Geneworks) were added to tough tubes containing samples in $300 \mu \mathrm{L}$ methanol 300 and samples were homogenized with a bead homogenizer (FastPrep-24, MP 301 Biomedical) at $6 \mathrm{~m} / \mathrm{s}$ for $40 \mathrm{~s}$ and transferred to new tubes. Beads were rinsed in 100 $302 \mu \mathrm{L}$ of methanol, which was added to the sample in new tubes. Samples were vortexed 303 with $1 \mathrm{~mL}$ of methyl-tert butyl ether at $4^{\circ} \mathrm{C}$ for $1 \mathrm{~h}$. Phase separation was induced by 304 adding $300 \mu \mathrm{L}$ of $150 \mathrm{mM}$ ammonium acetate (liquid chromatography-MS grade, 305 Fluka), vortexing for $5 \mathrm{~min}$ and centrifuging at 2,000 $\times \mathrm{g}$ for $5 \mathrm{~min}$. The upper organic 306 layer (representing around $800 \mu \mathrm{L}$ ) was transferred to a $2 \mathrm{~mL}$ glass vial and stored at $307-20^{\circ} \mathrm{C}$. Prior to MS analysis, each sample was diluted into methanol:chloroform (2:1 $308 \mathrm{v} / \mathrm{v}$ ) with $5 \mathrm{mM}$ ammonium acetate.

309 An aliquot of each extract was hydrolyzed to remove acyl-linked lipids and re310 extracted to improve mass spectrometric analysis of sphingolipids. Two hundred $\mu \mathrm{L}$ 311 of extract was added to $60 \mu \mathrm{L}$ of methanol containing $0.01 \%$ butylated 312 hydroxytoluene, $22 \mu \mathrm{L}$ of $10 \mathrm{M} \mathrm{NaOH}$ was added (final concentration $0.7 \mathrm{M}$ ), and 313 vortexed at 800 rounds per minute on an Eppendorf Mixmate for $2 \mathrm{~h}$ at room 314 temperature. Sixty $\mu \mathrm{L}$ of $150 \mathrm{mM}$ aqueous ammonium acetate was added to induce 315 phase separation. Tubes were vortexed and spun at $20,000 \times g$ for 5 min to complete 316 phase separation. The upper organic layer was removed to a new $2 \mathrm{~mL}$ glass vial, and 317 diluted into methanol:chloroform $(2: 1 \mathrm{v} / \mathrm{v})$ containing $5 \mathrm{mM}$ ammonium acetate prior 318 to mass spectrometric analysis.

319 Mass spectra were obtained with a chip-based nanoESI source (TriVersa Nanomate, 320 Advion) and a hybrid linear ion-trap-triple quadrupole MS (QTRAP 5500, 321 ABSCIEX). Ten $\mu \mathrm{L}$ of each sample extract in a sealed Eppendorf Twin-Tec 96 well 322 plate was aspirated and delivered to the MS through a nanoESI chip. Positive ion and 
323 negative ion acquisition was obtained as described previously (Tran et al. 2016).

324 Data smoothing, lipid identification, removal of isotope contribution from lower mass 325 species and correction for isotope distribution was performed in LipidView 326 (ABSCIEX) software version 1.2. A signal to noise ratio threshold of 20 was applied 327 for inclusion of ionized lipids. Extraction and solvent blanks were analyzed in each 328 data acquisition batch to exclude chemical or solvent impurities. Lipids were 329 quantified in LipidView by comparing peak area of each lipid to its class-specific 330 internal standard after isotope correction. Where odd-chain fatty acid phospholipids or 331 ether-linked phospholipids could not be distinguished, phospholipids were assumed to 332 be ether linked. A correction factor of 3.45 was applied to all ether-PE species to 333 account for the $29 \%$ difference in efficiency in the neutral loss of the $141 \mathrm{Da}$ 334 fragment from plasmenyl and diacyl PE respectively (Mitchell et al. 2007; Abbott et 335 al. 2013). Lipid species are annotated as per Liebisch et al. (2013) shorthand, except 336 for DAG and TAG.

337 LipidView data was exported to Excel then imported to Markerview v1.2.1.1 338 (Applied Biosystems, MDS Sciex) with lipid annotation and quantification for 339 statistical analysis of all individual lipid species. Pareto scaling without weighting was 340 applied for unsupervised mode principal component analysis. Multiple t-tests for 341 volcano plots were also performed in MarkerView (MDS Sciex). Grouping into lipid 342 classes and calculation of lipid proportions were performed in Microsoft Excel. 343 GraphPad Prism 7 was used to prepare graphs and perform ANOVA with multiple 344 comparisons on lipid class abundance. 


\section{Results}

348 The lipid composition of infected RBC is characteristic of the parasite's sex and

349 lifecycle stage

350 The lipidomic analysis was performed on uninfected RBC and RBC infected with 351 male gametocytes, female gametocytes or mature asexual blood stage parasites. Over 352230 lipid species were identified in the samples (for a complete list refer to 353 Supplementary Table 4). We performed a principle component analysis to determine 354 the relative contribution of each of these lipid species to the variation between 355 samples (Figure 1). The principal component analysis plot (Figure 1A) groups 356 together samples of similar lipid composition and the corresponding loading plot 357 (Figure 1B) illustrates which lipid species distinguish sample groups.

358

359 In considering all measured lipids by principal component analysis, the biggest 360 difference in lipid composition distinguishes infected from uninfected RBC regardless 361 of parasite lifecycle stage (principal component (PC) 1, Figure 1A). A third of the 362 lipid variation between samples nevertheless separates asexual parasite-infected RBC, 363 male gametocyte-infected RBC and female gametocyte-infected RBC (principal 364 component (PC) 2, Figure 1A). Parasite stages mainly cluster based on cholesteryl 365 ester (CE) species, free cholesterol (FC), diacylglycerol (DAG) and 366 phosphatidylcholine (PC) (Figure 1B).

\section{Female gametocyte infected RBC contain more neutral lipids than other stages}

369 Upon infection, total lipid abundance in infected RBC increases 3-5 fold and in sexual 370 stages, female gametocyte-infected RBC accumulate significantly more lipids than 371 male gametocyte-infected RBC (Figure 1C). However, this increase is not reflected 372 across all lipids: of the four main lipid categories (phospholipids, sphingolipids, free 373 cholesterol and neutral lipids), only neutral lipids are significantly more abundant in 374 female gametocyte-infected RBC compared to male gametocyte-infected RBC 375 (Figure 1C). This is also reflected in the relative proportions of lipid groups in each 376 stage: neutral lipids account for $31 \%$ of lipids in female gametocyte-infected RBC 377 compared to only $13 \%$ in male gametocyte-infected RBC (Figure 1D). Sphingolipid 378 and free cholesterol contents are similar in abundance but decreases in relative terms 379 in RBCs upon infection or in sexual stages. Phospholipids on the other hand are more 
380 abundant and represent a larger proportion to the relative lipid contribution of infected

381 RBCs.

382

383 In general, neutral lipids are a cellular means of storing energy and contribute to 384 intracellular signalling pathways. The nature of neutral lipids in asexual parasite385 infected RBC contrasts to that of gametocyte-infected RBC (Figure 2). Of the three 386 neutral lipids groups (cholesteryl ester (CE), diacylglycerol (DAG) and triacylglycerol 387 (TAG)), DAG and TAG are the predominant neutral lipids in asexual parasite388 infected RBC, whereas CE dominates the neutral lipid profile of gametocyte-infected $389 \mathrm{RBC}$ and uninfected RBC (Figure 2A and B). The proportions of neutral lipid categories are highly dynamic upon infection with asexual or sexual stage $P$. falciparum. In addition, accumulation of neutral lipids is sex-specific: female gametocyte-infected RBC accumulate significantly more CE than male gametocyteinfected RBC (Figure 2A). These trends are also observed at the individual lipid species level (Figure 2C and D). CE species characterise female gametocyte-infected $\mathrm{RBC}$ while asexual parasite-infected $\mathrm{RBC}$ are distinguished by DAG and TAG species (Figure 2C and D).

Phospholipids of gametocyte-infected RBC differ from those of asexual stage parasite infected RBC but are not sex-specific

Phospholipids are the main component of membranes and amongst others are required for organelle biogenesis. Hence it is not surprising that most lipids in iRBC belong to this group (Figure 1A). Although male gametocyte-infected RBC have a higher proportion of phospholipids (61\% phospholipids in males compared to $44 \%$ in females, Figure 1D), phospholipid abundance is similar in male and female gametocyte-infected RBC (Figure 1C). There were no significant differences in major phospholipid groups phosphatidyl choline (PC), phosphatidyl ethanolamine (PE), phosphatidyl serine (PS) or phosphatidyl glycerol (PG) between sexes (Figure 3A). PG is only detected in iRBC. Male gametocyte-infected RBC contain slightly more PC but slightly less PE than female gametocyte-infected RBC, both in terms of

412 Principal component analysis based only on phospholipids does not distinguish 413 between male and female gametocyte-infected RBC (Figure 3C). However, 
414 gametocyte-infected RBC cluster apart from uninfected RBC and asexual parasite-

415 infected RBC mostly due to PE species (Figure 3C and D). Overall, male and female

416 gametocyte-infected RBC phospholipids are similar to each other, but distinct from

417 host cell and asexual parasite-infected RBC phospholipids.

418

The sphingolipid dihydrosphingomyelin is a key characteristic of female

421 Sphingolipids are also components of cell membranes and form detergent-resistant

422 lipid domains that are platforms for intracellular signalling. Like phospholipids,

423 overall sphingolipid abundance is not significantly different between male and female

424 gametocyte-infected RBC (Figure 1C). However, breaking down sphingolipids into

425 its three main groups (sphingomyelin (SM), dihydrosphingomyelin (DHSM) and

426 ceramide) reveals gametocyte and sex-specific sphingolipid groups (Figure 4). Unlike

427 the abundance of the major sphingolipid SM that is similar in all samples (Figure 4A),

428 gametocyte-infected RBC (especially females) accumulate DHSM (Figure 4A and B).

430 Principal component analysis of sphingolipids alone does not distinguish uninfected 431 RBC from asexual parasite-infected RBC. However, male and female gametocyte432 infected RBC cluster separately (Figure 4C). DHSM 20:0, 16:0 and 18:0 especially 433 distinguish gametocyte-infected RBC from the other samples (Figure 4D). Overall, 434 accumulation of DHSM marks gametocytogenesis and is even more pronounced in 435 females.

437 Of the lipid species with more than a two-fold difference between female and male 438 gametocyte-infected RBC, six are significantly more abundant in female gametocyte439 infected RBC (Figure 5A). Five of these are saturated DHSM species (between C16440 C20 in size), the other is SM 22:2, identifying sphingolipids as the major sex-specific 441 lipid group. On average several neutral lipid species also appear more abundant in 442 female gametocytes, however this difference lacks statistical significance due to 443 variation between biological replicates. No lipid species are significantly more 444 abundant in male gametocyte-infected RBC, although some phospholipid species are 445 more abundant on average. Overall, the sex-specific lipid profile of gametocyte446 infected RBC points towards DHSM as a key characteristic of female gametocytes. 
448 Compared to asexual stage parasite-infected RBC, both sexes of gametocyte-infected 449 RBC contain more DHSM 20:0, 18:0 and 16:0 (Figure 5B \& C). Asexual parasite450 infected RBC on the other hand contain significantly more of some PC species 451 compared to both sexes of gametocyte-infected RBC (PC32:0, PC32:1, PC34:0, 452 PC36:0 [in males only]). CE 16:1 and CE 16:0 are the only lipid species that are more 453 abundant in female gametocyte-infected RBC but not in males when compared to 454 asexual parasite-infected RBC, which might point to specialised functions of these 455 lipid species in female parasites. Several phospholipid and sphingolipid species are 456 significantly more abundant in each sex of gametocyte-infected RBC compared to 457 uninfected RBC (Figure 5D \& E). Overall, gametocytes significantly modify the lipid 458 profile of the host $\mathrm{RBC}$ during maturation in a manner that distinguishes them from 459 asexual parasites.

460

Sphingolipid synthesis is functionally important in both asexual and sexual blood stages

463 To investigate the functional significance of the sex-specific lipid composition, 464 gametocytes were exposed to three compounds that block the synthesis of the most 465 sex specific lipids (CE and sphingolipids) from day 2 to 6 post commitment (Figure 466 6). Thereafter the sex-specific viability of gametocytes was measured by flow cytometry. GT11 is an analogue of dihydroceramide that cannot be metabolised by 468 dihydroceramide desaturase in mammalian cells, thereby blocking the enzyme responsible for ceramide synthesis (Bedia et al. 2005). At concentrations greater that 5 $\mu \mathrm{M}$, GT11 more generally decreased de novo sphingolipid synthesis in cultured mammalian cells (Triola et al. 2004). Glibenclamide and Sandoz 58-035 directly inhibit cholesterol esterification by acyl-coenzyme A: cholesterol acyltransferase in

473 mammalian cells (Ross et al. 1984). At $10 \mu \mathrm{M}$ only the sphingolipid synthesis

474 inhibitor GT11 appears to impact gametocyte viability in a male-specific manner 475 (Figure 6).

477 The apparent sex-specific function of sphingolipid synthesis in gametocytes was 478 further investigated by reverse genetics. DHSM and SM are synthesised by 479 sphingomyelin synthase (SMS) from dihydroceramide and ceramide respectively. $P$. 480 falciparum encodes two such enzymes (SMS1 and SMS2) that are expressed in a 
481 parasite stage- and sex-specific manner. SMS activity contributes to the synthesis of

482 SM, a component of detergent-resistant lipid domains in cell membranes, but also

483 catabolises the intracellular signalling molecule ceramide.

484

485 Three independent attempts to disrupt both SMS1 and SMS2 genes together did not 486 yield any parasites after transfection. However, disruption of each SMS gene 487 individually produced asexual blood stage parasites that could be analysed further 488 (Supplementary Figures 1 and 2). We first tested the relative fitness of these knock 489 out (KO) parasites. In 28-day co-cultures wild type parasites quickly outgrew each of 490 the KO parasites (Figure 7), suggesting that SMS1 and SMS2 are each required for 491 optimal asexual parasite proliferation. Depleting the culture medium lipids by $75 \%$ 492 did not exacerbate the growth defect observed in each KO cell line (Figure 7). In other 493 words, SMS disruption is not readily compensated by an increase in extra-cellular 494 lipid scavenging. Treatment with the sphingolipid synthesis inhibitors GT11 had the 495 same effect on WT and each KO cell line (Figure 8). This suggests that GT11, an 496 analogue of dihydroceramide, does not block SMS in asexual blood stage parasites.

497 Given that sphingolipid composition is distinctive in gametocytes, the impact of 498 disrupting each SMS was next investigated in sexual blood-stage parasites. The 499 absence of either SMS1 or SMS2 increased gametocyte commitment compared to WT 500 parasites (Figure 8A and B). This may result from asexual blood-stage parasite stress 501 given that gametocyte commitment is a generic parasite stress response (Dixon et al. 502 2008). When treated with GT11 however, gametocytes lacking a SMS were 503 hypersensitive compared to WT gametocytes (Figure 8C). This suggests that SMS and 504 dihydroceramide desaturase function is additive in gametocytes, especially in 505 conditions where SMS function is limited either through disruption of one of the SMS 506 genes (Figure 8C) or by native reduction of SMS gene expression in males relative to 507 females (Figure 6).

508 


\section{Discussion}

511 In summary the lipid composition and abundance of lipids in $P$. falciparum 512 gametocytes is sex-specific. Male/female gametocyte lipid dimorphism precedes 513 gametocyte activation and reflects their sex-specific functions.

515 Female gametocyte-infected RBC stockpile the neutral lipid CE but were resistant to 516 CE synthesis inhibitors Sandoz 58-035 and Glibenclamide. This suggests that acyl517 coenzyme A: cholesterol acyltransferase -mediated CE synthesis is not essential in 518 mature gametocytes. CE could instead be synthesised by lecithin-cholesterol 519 acyltransferase, or could be scavenged from the host. Alternatively, CE may only be 520 required for later developmental stages. Indeed the lipids in the fertilised zygote are 521 exclusively contributed by the female gametocyte. While the zygote develops in the 522 midgut lumen, neutral lipids are diverted from the blood meal to the eggs of the 523 mosquito via lipophorin (Costa et al. 2018). The zygote is therefore vulnerable to lipid 524 depletion in the mosquito midgut lumen, and may depend on maternal CE reserves at 525 this lifecycle stage. In particular, given that mosquitoes are incapable of cholesterol 526 synthesis (Clayton 1964), CE could be a source of cholesterol for the parasite in 527 mosquito stages. P. falciparum encodes esterases that could potentially release 528 cholesterol from CE stores (Butler et al. 2020). Neutral lipid staining of the female 529 gametocyte previously highlighted a large neutral lipid body in the cytoplasm (Tran et 530 al. 2014). Potentially the female specific CE store is amassed by the putative lipid 531 transporter gABCG2. Hence, it would be interesting to test the effect of CE synthesis 532 inhibitors on the mosquito stages in future studies.

533

534 Male gametocyte-infected RBC do not contain more phospholipids than female 535 gametocyte-infected RBC. Phospholipids are the main component of membranes and 536 are likely required for the rapid cell divisions during male gametocyte activation when 537 eight microgametes are formed within the male gametocyte. While additional plasma 538 membranes may not be required in females, female gametocytes contain an elaborate 539 mitochondrial network bound by a double membrane rich in phospholipids, as well as 540 abundant membrane bound osmiophilic bodies (Langreth et al. 1978; Jensen 1979;

541 Sinden 1982; Ponnudurai et al. 1986). Although the total phospholipid content of 542 male and female gametocyte-infected RBC is similar, the allocation of phospholipids 543 between organelles may be sex-specific. 
544 For example, PG serves as the precursor for cardiolipin found exclusively in the 545 mitochondria (Gebert et al. 2009; de Kroon et al. 1999; Daum 1985). Consistent with 546 this, PG is absent in uninfected RBC, which lack mitochondria. Surprisingly however, 547 there is no difference in PG between male and female gametocytes despite female 548 gametocytes relying more on their mitochondria than males. Indeed mitochondrial 549 proteins have been shown to be more abundant in female than in male gametocytes 550 (Miao et al., 2017). Female gametes in the mosquito have an increased energy 551 demand (probably in anticipation of the post-fertilisation stage (MacRae et al. 2013), 552 whereas male gametocytes lose their mitochondria completely during the 553 development into microgametes (Okamoto et al. 2009). The presence of comparable 554 amounts of PG both in male and female iRBC argues for a similar number or size of 555 mitochondria at least at the analysed stage of development (stage IV gametocytes). 556 However, less PG is detected in the RBC infected with sexual stages compared to the 557 RBC infected with asexual stages despite the upregulation of tricarboxylic acid cycle 558 function in gametocytes (MacRae 2013). This cautions against the simple correlation 559 between PG and mitochondrial function. Nonetheless, the specificity and 560 accumulation of PG in the parasite could be targeted by antimalarial compounds 561 effective against both asexual and sexual blood stage parasites.

562 Phospholipid composition distinguished both male and female gametocyte-infected 563 RBC from asexual parasite-infected RBC and uninfected RBC. Less phospholipids 564 were present in gametocyte-infected RBC compared to asexual parasite-infected RBC 565 consistent with asexual replication requiring phospholipid-rich membrane biogenesis 566 (e.g. for the plasma membrane of a growing parasites, for merozoite formation and for 567 RBC modifications such as Maurer's clefts). The increase in PS between trophozoites 568 and gametocytes reported by Gulati et al. (2015) was not observed in this study, 569 perhaps due to technical differences in sample preparation.

571 In asexual blood stages, reduction of sphingolipid metabolism by disruption of either 572 SMS1 or SMS2 was not lethal, but incurred a growth defect. Disruption of both SMS 573 genes on the other hand appears to be lethal for asexual blood stage parasites, despite 574 SMS2 being expressed at very low levels in this lifecycle stage. This suggests that $d e$ 575 novo sphingomyelin synthesis is essential in asexual blood stages and indicates that 576 each SMS is - to a certain degree - functionally redundant. Surprisingly this defect 577 was not exacerbated by depleting the culture medium lipids by $75 \%$, or by inhibiting 
578 dihydroceramide desaturase with GT11. This supports the hypothesis that SMS

579 activity regulates intracellular ceramide concentration rather than sphingomyelin itself

580 being essential for asexual blood stage proliferation. Indeed, ceramide might

581 otherwise build up to cytotoxic levels due to sphingomyelinase activity, which has

582 previously been described in asexual stages (Hanada et al. 2002). Inhibition of

583 ceramide production by sphingomyelinase with GW4869 is also lethal for asexual $P$.

584 falciparum (Gulati et al. 2015), further illustrating the importance of regulating

585 ceramide abundance.

586

587 The most distinctive feature of gametocyte-infected RBC, particularly female 588 gametocyte-infected RBC, is the presence of DHSM species. This is consistent with 589 other studies that suggest de novo sphingolipid synthesis, rather than host SM 590 hydrolysis by neutral sphingomyelinase, is active in gametocytes (Gulati et al. 2015; 591 Tran et al. 2016). Here we sought to test whether DHSM accumulation in 592 gametocytes results from decreased catabolism by dihydroceramide desaturase and/or 593 from increased synthesis by SMS. Inhibition of dihydroceramide desaturase with 10 $594 \mu \mathrm{M}$ GT11 selectively killed male gametocytes (Figure 6) suggesting that 595 dihydroceramide desaturase activity is sex-specific, which may contribute to the 596 difference in DHSM abundance. The male-specific effect of GT11 was not observed 597 in combined male and female gametocytes (Figure 8), presumably due to the 598 gametocyte population being female biased. However, gametocytes that lack an SMS 599 were hypersensitive to GT11 (Figure 8). In other words, reduced SMS activity (due to 600 endogenous differential SMS gene expression between male and female gametocytes 601 or through SMS gene disruption) appears to make parasites more susceptible to 602 dihydroceramide desaturase inhibition by GT11. Given that dihydroceramide 603 desaturase and SMS share dihydroceramide as a substrate, hypersensitivity to GT11 in 604 gametocytes with reduced SMS activity may result from an accumulation of 605 dihydroceramide. Conversely, accumulation of DHSM in gametocytes may be a 606 means of reducing intracellular dihydroceramide levels.

607 Similarly, the observed increased gametocyte commitment in SMS KO cell lines 608 could be a stress response to elevated dihydroceramide concentrations. Indeed it has 609 recently been observed that dihydroceramides are biologically active lipids involved 610 in diverse mammalian cell functions (Siddique et al. 2015). Lipids have previously 611 been implicated in inducing gametocytogenesis in P. falciparum (Brancucci et al. 
612 2017). In particular, depletion of serum lysophosphatidylcholine, a precursor of PC

613 synthesis, is known to trigger gametocytogenesis. We observed no significant

614 difference in lysophosphatidylcholine abundance between male and female

615 gametocytes (supplementary figure S3). PC is a substrate of SMS-mediated SM and

616 DHSM synthesis. As such, reducing parasite SMS activity may have mimicked the

617 downstream effect of PC depletion and triggered gametocytogenesis.

618

619 Overall, this study has shown that lipid metabolism is not only variable between 620 parasite lifecycle stages, but is also sex-specific. These differences have implications

621 for the effectiveness of drugs targeting metabolic pathways, and should be considered

622 in the design of future transmission blocking antimalarial treatments. In terms of the 623 fundamental biology of the parasite, the lipid profile of gametocytes is a testament to 624 the parasite's ability to thrive in two radically different host environments. The 625 lipidome presented here provides a reference for unravelling the complex sex-specific 626 function of lipids in the P. falciparum.

627

628 Acknowledgements:

629 FACS was performed with assistance from Dr. Harpeet Vohra and Mr. Michael 630 Devoy. We are grateful to the Australian Red Cross for providing human red blood 631 cells and serum. Funding was provided by the Australian Research Council 632 (DP180103212) and the National Health and Medical Research Council of Australia 633 (APP1182369). M.C.R. is supported by the Australian Government Research Training 634 Program Scholarship and The Australian National University.

635

636

638 640 641

\section{Author contributions:}

M.C.R. and A.G.M. conceived and designed the study; M.C.R., P.T., S.B. and D.C. performed the experiments; M.C.R., S.B., D.C., T.M. and A.G.M. analysed the data; and M.C.R. and A.G.M. wrote the paper. All authors contributed to the editing of the paper. 
643 Conflict of Interest Disclosure:

644 The authors declare that they have no conflict of interest.

645

646 Submission of data set to database:

647 The mass spectrometry raw data of the lipidomics experiments have been deposited in

648 the MetaboLights database (http://www.ebi.ac.uk/metabolights).

649

650

\section{Figure legends:}

652 Figure 1: A-B: Principal component analysis (A) and loading plot (B) of the lipidome 653 of uninfected red blood cells (uRBC- red) and red blood cells infected with asexual 654 stage parasites (Asexual- green), female gametocytes (Female - pink) and male 655 gametocytes (Male - blue). Black circles in A indicate sample groups. The individual 656 lipid species are colour-coded to indicate the group they belong to (Neutral lipids 657 blue; Phospholipids - red, Sphingolipids - green, Free Cholesterol - indigo). C-D:

658 Mean lipid abundance with standard deviation (C) and proportions (D) of lipid classes 659 in uninfected red blood cells ( $\mathrm{URBC}$ ) and red blood cells infected with asexual stage 660 parasites (Asexual), male gametocytes (Male) and female gametocytes (Female).

661 Results averaged from three independent biological replicates of $10^{7}$ cells each.

662 Significance calculated by ANOVA. ***: $\mathrm{p}<0.001, *: \mathrm{p}<0.1$, ns: not significant, $\mathrm{p}>$

663 0.1. PC1 and 2: first and second principal component; CE: cholesteryl ester; FC: free 664 cholesterol; PC: phosphatidylcholine; PE: phosphatidylethanolamine; DAG: 665 diacylglycerol. 
668 Figure 2: A-B: Mean neutral lipid abundance with standard deviation (A) and mean 669 proportions (B) of uninfected red blood cells (URBC) and red blood cells infected 670 with asexual stage parasites (Asexual), male gametocytes (Male) or female 671 gametocytes (Female). Results averaged from three independent biological replicates 672 of $10^{7}$ cells. Significance calculated by ANOVA. ****: $\mathrm{p}<0.0001$, ns: not significant, 673 p> 0.1. CE: cholesteryl ester; DAG: diacylglycerol; TAG: triacylglycerol. C-D: 674 Principal component analysis (C) and loading plot (D) of neutral lipid composition of 675 uninfected red blood cells ( $\mathrm{URBC}$ ) and red blood cells infected with asexual stage 676 parasites (Asexual), male gametocytes (Male) or female gametocytes (Female). PC1

677 and 2: first and second principal component; CE: cholesteryl ester; DAG: 678 diacylglycerol; TAG: triacylglycerol.

681 Figure 3: A-B: Mean phospholipid composition with standard deviation (A) and 682 mean proportions (B) of uninfected red blood cells ( $\mathrm{URBC}$ ) and red blood cells 683 infected with asexual stage parasites (Asexual), male gametocytes (Male) or female 684 gametocytes (Female). Results averaged from three independent biological replicates 685 of $10^{7}$ cells. Significance calculated by two-way ANOVA. ns: not significant, p>0.1. 686 C-D: Principal component analysis (C) and loading plot (D) of phospholipid 687 composition of uninfected red blood cells ( $\mathrm{URBC}$ ) and red blood cells infected with 688 asexual stage parasites (Asexual), male gametocytes (Male) or female gametocytes 689 (Female). PC1 and 2: first and second principal component; PC: phosphatidylcholine; 690 PE: phosphatidylethanolamine; PS: phosphatidylserine; PG phosphatidylglycerol. 
693 Figure 4: A-B: Mean sphingolipid abundance with standard deviation (A) and mean

694 proportion (B) in uninfected red blood cells (uRBC) and red blood cells infected with

695 asexual stage parasites (Asexual), male gametocytes (Male) or female gametocytes

696 (Female). Results averaged from three independent biological replicates of $10^{7}$ cells.

697 Significance calculated by two-way ANOVA. **: $\mathrm{p}<0.01$, ns: not significant, $\mathrm{p}>0.1$.

698 C-D: Principal component analysis (C) and loading plot (D) of sphingolipid

699 composition of uninfected red blood cells (uRBC) and red blood cells infected with

700 asexual stage parasites (Asexual), male gametocytes (Male) or female gametocytes

701 (Female). PC1 and 2: first and second principal component; DHSM:

702 dihydrosphingomyelin; SM: sphingomyelin; Cer: ceramide.

703

704

705 Figure 5: Volcano plot of lipid species in male and female gametocyte-infected red

706 blood cells. A, C and E: Comparison of female gametocyte-infected red blood cell

707 lipids to male gametocyte-infected red blood cells (A), asexual-stage infected red

708 blood cells (C) and uninfected red blood cells (E). B and D: Comparison of male

709 gametocyte-infected red blood cell lipids to asexual-stage infected red blood cells (B)

710 and uninfected red blood cell (RBC) (D). Fold change averaged from three biological

711 repeats of $10^{7}$ cells each. $\mathrm{P}$ values calculated by student $\mathrm{t}$ tests. Grey area represents

712 changes that are less than two-fold different between samples and/or have a

713 significance of $\mathrm{p}>0.01$. Circles in A highlight groups of lipids. DAG: diacylglycerol;

714 DHSM: dihydrosphingomyelin; CE: cholesteryl ester; PC: phosphatidylcholine; PE:

715 phosphatidylethanolamine; PS: phosphatidylserine; SM: sphingomyelin; TAG:

716 triacylglycerol. 
719 Figure 6: A: Gametocyte viability following chemical inhibition of sphingolipid or

720 CE synthesis. Sphingolipid synthesis was disrupted with $10 \mu \mathrm{M}$ GT11 and CE

721 synthesis was inhibited with $10 \mu \mathrm{M}$ Sandoz 58-035 or $10 \mu \mathrm{M}$ glibenclamide from day

7222 to 6 post-commitment. For each gametocyte sex, results are normalised to viability

723 following exposure to the DMSO solvent control and $100 \mu \mathrm{M}$ artemisinin. Average

724 result and standard deviation presented from three biological replicates, each

725 performed in technical triplicates. B: diagram of GT11 inhibition of sphingolipid

726 synthesis. DHCer: dihydroceramide; DHSM: dihydrosphingomyelin; SMS:

727 sphingomyelin synthase; Cer: ceramide; SM: sphingomyelin.

728

729

730 Figure 7: Fitness competition between 3D7 WT and SMS1 KO (A) or SMS2 KO

731 clones (B) in complete (CCM, continuous line) and lipid-depleted culture medium

732 (ICM, dashed line) over 28 days. On day 0 equal amounts of 3D7 WT and SMS1 KO

733 clone E6 (blue, A), SMS1 KO clone E10 (red, A), SMS2 KO clone C9 (green, B) or

734 SMS2 KO clone D4 (black, B) were combined in a co-culture. The proportion of each

735 genotype in the culture was monitored on day 0,12 and 28 by qPCR of the hDHFR

736 resistance cassette (to detect KO genotype) and the disrupted section of the SMS1 or 2

737 genes (to detect WT genotype). At some time points technical problems inhibited

738 monitoring the culture so measurements were performed 4 days afterwards instead.

739 Results from 2-4 independent biological repeats are shown as mean and standard

740 deviation. Statistical significance between day 0 and day 28 was calculated by two-

741 way ANOVA. In B results in ICM were not significant (ns), whereas p<0.001 (***) 
742 for WT vs SMS1 clone E10 in ICM in A and p<0.0001 (****) for all other results in 743 A and B.

744

745

746 Figure 8: Gametocytaemia in 3D7 WT and SMS1 KO (A) or SMS2 KO clones (B)

747 was monitored every second day from day 2 to day 8 post commitment and compared

748 to WT gametocytaemia on day 2. (C) Effect of $10 \mu \mathrm{M} \mathrm{GT11}$ on the commitment to

749 gametocytaemia in 3D7 WT, SMS1 KO and SMS2 KO clones. The cultures were

750 treated with GT11 $(10 \mu \mathrm{M})$ or $0.1 \%$ DMSO (control) for 72 hours from day 3 to day 0

751 pre-commitment. Gametocytaemia was determined on day 8 and compared to

752 gametocytaemia of the untreated WT culture. Gametocytaemia is presented as mean

753 and standard error of mean from three biological replicates, each performed in

754 technical triplicates. Significance calculated by unpaired t-test. ns: not significant,

$755 p>0.1, *: p<0.05, * *: p<0.01$.

756

757

758 Figure 9: Overview of lipid changes in P. falciparum during asexual and sexual

759 blood stages. Grey background: graphic representation of uninfected RBC, RBC

760 infected with trophozoite parasites and male and female infected RBCs. Dashed arrow

761 indicate that there is no direct development from trophozoites to sexual stages and the

762 intermediate stages are not shown. Blue background: neutral lipids; red background:

763 phospholipids; green background: sphingolipids; yellow background: free cholesterol.

764 The left column shows graphic representations of the lipid groups and their

765 abreviation. The red arrows indicate changes relative to the previous life-cycle stage.

766 Upward arrow means >3-fold increase; horizontal arrow means moderate or no 
767 changes (changes that are more than 0.5 -fold and less than 2-fold); a declining arrow

768 indicates decrease of less than half-fold and a downward arrow signifies a decrease of

769 more than 0.3 of the previous value. The male and female infected RBCs are relative

770 to the trophozoite-infected RBCs. Stars indicate significant changes between male and

771 females. CE: cholesteryl ester; DAG: diacylglycerol; TAG: triacylglycerol; PC:

772 phosphatidylcholine; PE: phosphatidylethanolamine; PS: phosphatidylserine; PG:

773 phosphatidylglycerol; DHSM: dihydrosphingomyelin; SM: sphingomyelin. Cer:

774 ceramide; FC: free cholesterol.

775

776 


\section{References:}

Abbott, S.K. et al., 2013. An improved high-throughput lipid extraction method for the analysis of human brain lipids. Lipids, 48(3), pp.307-318.

Aikawa, M. et al., 1984. New observations on gametogenesis, fertilization, and zygote transformation in Plasmodium gallinaceum. The Journal of protozoology, 31(3), pp.403-413.

Bedia, C. et al., 2005. Analogs of the dihydroceramide desaturase inhibitor GT11 modified at the amide function: synthesis and biological activities. Organic \& biomolecular chemistry, 3(20), pp.3707-3712.

Bennink, S., Kiesow, M.J. \& Pradel, G., 2016. The development of malaria parasites in the mosquito midgut. Cellular microbiology, 18(7), pp.905-918.

Brancucci, N.M.B. et al., 2017. Lysophosphatidylcholine regulates sexual stage differentiation in the human malaria parasite Plasmodium falciparum. Cell, 171(7), pp.1532-1544.e15.

Butler, J.H. et al., 2020. Resistance to some but not other dimeric lindenane sesquiterpenoid esters is mediated by mutations in a Plasmodium falciparum esterase. ACS infectious diseases, 6(11), pp.2994-3003.

Canning, E.U. \& Sinden, R.E., 1975. Nuclear organisation in gametocytes of Plasmodium and Hepatocystis: a cytochemical study. Zeitschrift für Parasitenkunde (Berlin, Germany), 46(4), pp.297-299.

Clayton, R.B., 1964. The utilization of sterols by insects. Journal of lipid research, 5, pp.3-19.

Costa, G. et al., 2018. Non-competitive resource exploitation within mosquito shapes within-host malaria infectivity and virulence. Nature communications, 9(1), p.3474.

Daum, G., 1985. Lipids of mitochondria. Biochimica et biophysica acta, 822(1), pp.1-42.

de Kroon, A.I. et al., 1999. Isolation and characterization of highly purified mitochondrial outer membranes of the yeast Saccharomyces cerevisiae (method). Molecular membrane biology, 16(2), pp.205-211.

Dixon, M.W.A. et al., 2008. Sex in Plasmodium: a sign of commitment. Trends in parasitology, 24(4), pp.168-175.

Dourmashkin, R.R., Dougherty, R.M. \& Harris, R.J., 1962. Electron microscopic observations on Rous sarcoma virus and cell membranes. Nature, 194, pp.1116-1119.

Fivelman, Q.L. et al., 2007. Improved synchronous production of Plasmodium falciparum gametocytes in vitro. Molecular and biochemical parasitology, 154(1), pp.119-123.

Fyrst, H. \& Saba, J.D., 2010. An update on sphingosine-1-phosphate and other sphingolipid mediators. Nature chemical biology, 6(7), pp.489-497.

Gardner, M.J. et al., 2002. Genome sequence of the human malaria parasite Plasmodium falciparum. Nature, 419(6906), pp.498-511.

Gebert, N. et al., 2009. Mitochondrial cardiolipin involved in outer-membrane protein biogenesis: implications for Barth syndrome. Current biology : $C B, 19(24)$, pp.2133-2139.

Gulati, S. et al., 2015. Profiling the essential nature of lipid metabolism in asexual blood and gametocyte stages of Plasmodium falciparum. Cell host \& microbe, 18(3), pp.371-381.

Hanada, K. et al., 2002. Plasmodium falciparum phospholipase C hydrolyzing sphingomyelin and lysocholinephospholipids is a possible target for malaria chemotherapy. The Journal of 
experimental medicine, 195(1), pp.23-34.

Jensen, J.B., 1979. Observations on gametogenesis in Plasmodium falciparum from continuous culture. The Journal of protozoology, 26(1), pp.129-132.

Lambros, C. \& Vanderberg, J.P., 1979. Synchronization of Plasmodium falciparum erythrocytic stages in culture. The Journal of parasitology, 65(3), pp.418-420.

Langreth, S.G. et al., 1978. Fine structure of human malaria in vitro. The Journal of protozoology, 25(4), pp.443-452.

Lasonder, E. et al., 2016. Integrated transcriptomic and proteomic analyses of $P$. falciparum gametocytes: molecular insight into sex-specific processes and translational repression. Nucleic acids research, 44(13), pp.6087-6101.

Liebisch, G. et al., 2013. Shorthand notation for lipid structures derived from mass spectrometry. Journal of lipid research, 54(6), pp.1523-1530.

López-Barragán, M.J. et al., 2011. Directional gene expression and antisense transcripts in sexual and asexual stages of Plasmodium falciparum. BMC genomics, 12(1), p.587.

MacRae, J.I. et al., 2013. Mitochondrial metabolism of sexual and asexual blood stages of the malaria parasite Plasmodium falciparum. BMC biology, 11(1), p.67.

Maier, A.G. \& Rug, M., 2013. In vitro culturing Plasmodium falciparum erythrocytic stages. Methods in molecular biology (Clifton, N.J.), 923(Chapter 1), pp.3-15.

Maier, A.G. et al., 2008. Exported proteins required for virulence and rigidity of Plasmodium falciparum-infected human erythrocytes. Cell, 134(1), pp.48-61.

Matyash, V. et al., 2008. Lipid extraction by methyl-tert-butyl ether for high-throughput lipidomics. Journal of lipid research, 49(5), pp.1137-1146.

Miao, J. et al., 2017. Sex-specific biology of the human malaria parasite revealed from the proteomes of mature male and female gametocytes. Molecular \& cellular proteomics : MCP, p.mcp.M116.061804.

Mitchell, T.W., Buffenstein, R. \& Hulbert, A.J., 2007. Membrane phospholipid composition may contribute to exceptional longevity of the naked mole-rat (Heterocephalus glaber): a comparative study using shotgun lipidomics. Experimental gerontology, 42(11), pp.1053-1062.

Okamoto, N. et al., 2009. Apicoplast and mitochondrion in gametocytogenesis of Plasmodium falciparum. Eukaryotic cell, 8(1), pp.128-132.

Pfaffl, M.W., 2001. A new mathematical model for relative quantification in real-time RT-PCR. Nucleic acids research, 29(9), p.e45.

Ponnudurai, T. et al., 1986. Synchronization of Plasmodium falciparum gametocytes using an automated suspension culture system. Parasitology, 93 ( Pt 2), pp.263-274.

Ridgway, M.C. et al., 2020. Novel method for the separation of male and female gametocytes of the malaria parasite Plasmodium falciparum that enables biological and drug discovery. mSphere, $5(4)$.

Ridgway, M.C., Cihalova, D. \& Maier, A.G., 2021. Sex-specific separation of Plasmodium falciparum gametocyte populations. Bio-protocol, 11(11), p.e4045.

Ross, A.C. et al., 1984. Selective inhibition of acyl coenzyme A:cholesterol acyltransferase by compound 58-035. The Journal of biological chemistry, 259(2), pp.815-819. 
859

860

861

862

863

864

865

866

867

868

869

870

871

872

873

874

875

876

877

878

879

880

881

882
Rug, M. \& Maier, A.G., 2013. Transfection of Plasmodium falciparum. Methods in molecular biology (Clifton, N.J.), 923(Chapter 6), pp.75-98.

Ruijter, J.M. et al., 2009. Amplification efficiency: linking baseline and bias in the analysis of quantitative PCR data. Nucleic acids research, 37(6), p.e45.

Siddique, M.M. et al., 2015. Dihydroceramides: from bit players to lead actors. The Journal of biological chemistry, 290(25), pp.15371-15379.

Sinden, R.E., 1982. Gametocytogenesis of Plasmodium falciparum in vitro: an electron microscopic study. Parasitology, 84(1), pp.1-11.

Sinden, R.E. et al., 1978. Gametocyte and gamete development in Plasmodium falciparum. Proceedings of the Royal Society of London. Series B, Biological sciences, 201(1145), pp.375399.

Sinden, R.E., Hartley, R.H. \& Winger, L., 1985. The development of Plasmodium ookinetes in vitro: an ultrastructural study including a description of meiotic division. Parasitology, 91 ( $\mathrm{Pt}$ 2), pp.227-244.

Tran, P.N. et al., 2014. A female gametocyte-specific ABC transporter plays a role in lipid metabolism in the malaria parasite. Nature communications, 5, p.4773.

Tran, P.N. et al., 2016. Changes in lipid composition during sexual development of the malaria parasite Plasmodium falciparum. Malaria journal, 15(1), p.73.

Triola, G. et al., 2004. Specificity of the dihydroceramide desaturase inhibitor N-[(1R,2S)-2-hydroxy1-hydroxymethyl-2-(2-tridecyl-1-cyclopropenyl)ethyl]octanamide (GT11) in primary cultured cerebellar neurons. Molecular pharmacology, 66(6), pp.1671-1678.

Vlachou, D. et al., 2004. Real-time, in vivo analysis of malaria ookinete locomotion and mosquito midgut invasion. Cellular microbiology, 6(7), pp.671-685. 

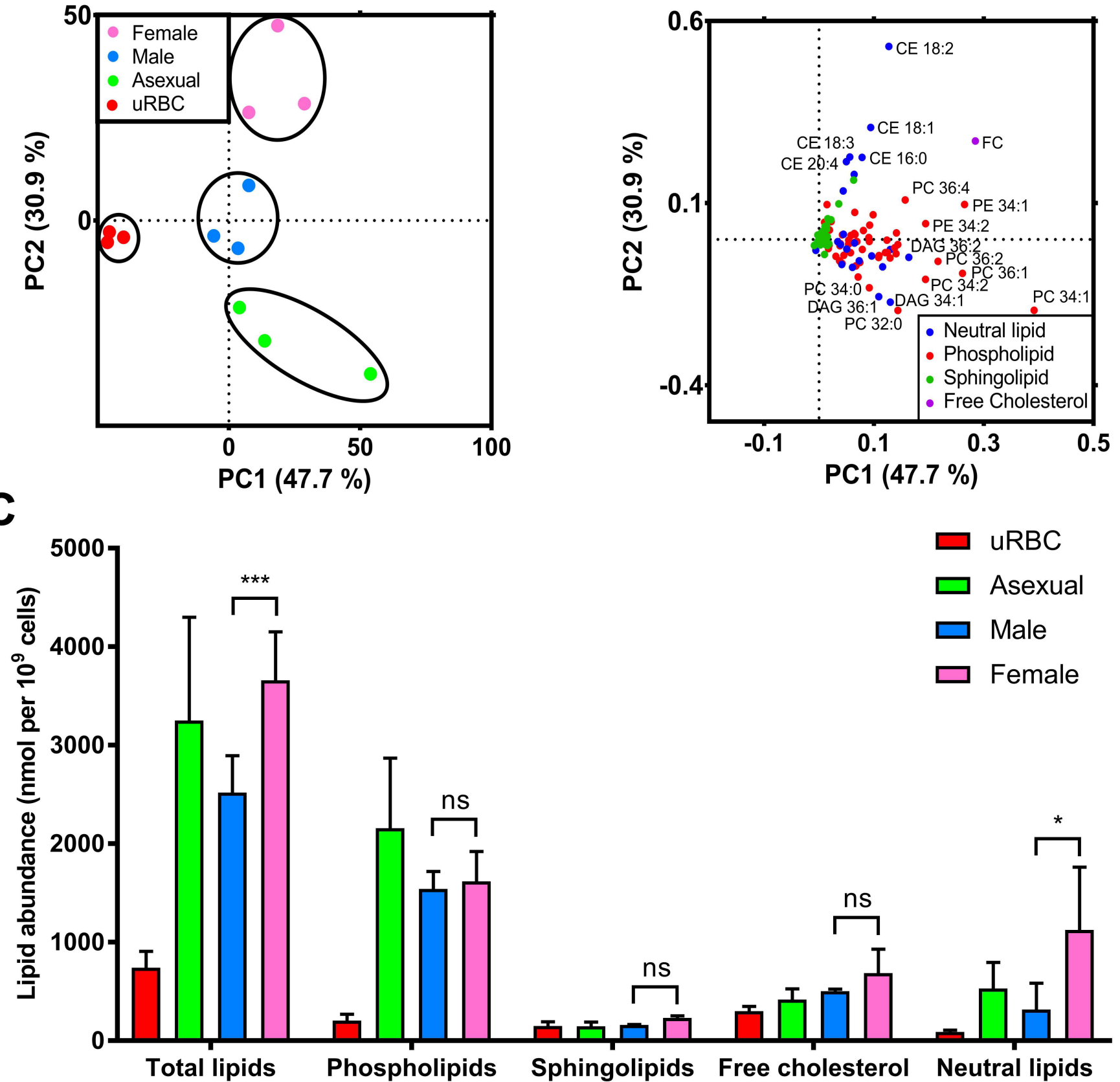

D uRBC

Asexual

Male

Female
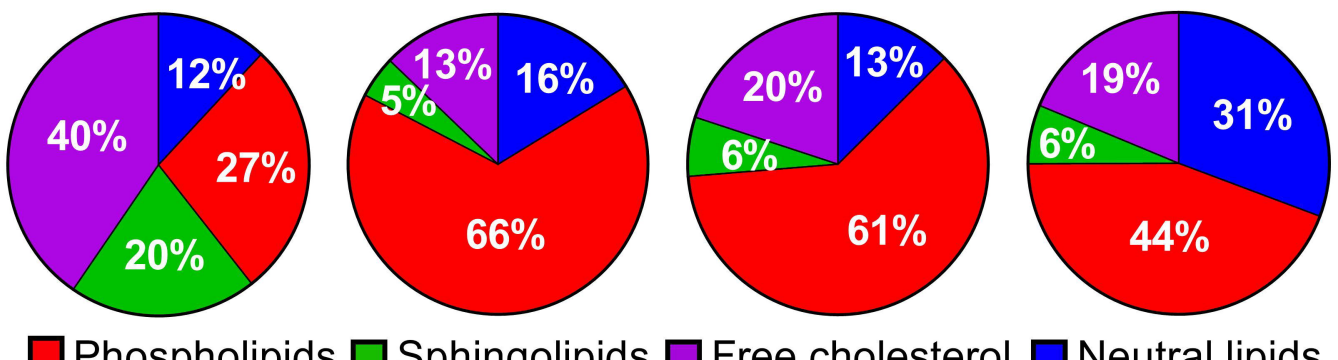

Phospholipids

Sphingolipids

Free cholesterol

Neutral lipids 
A

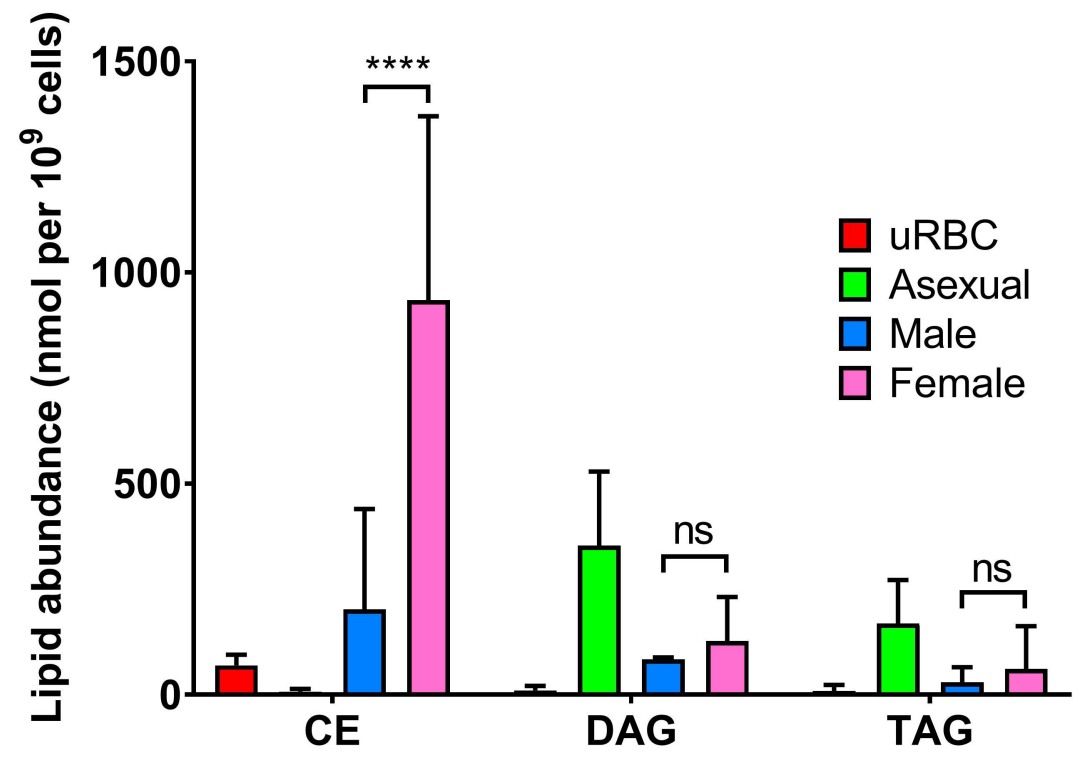

B
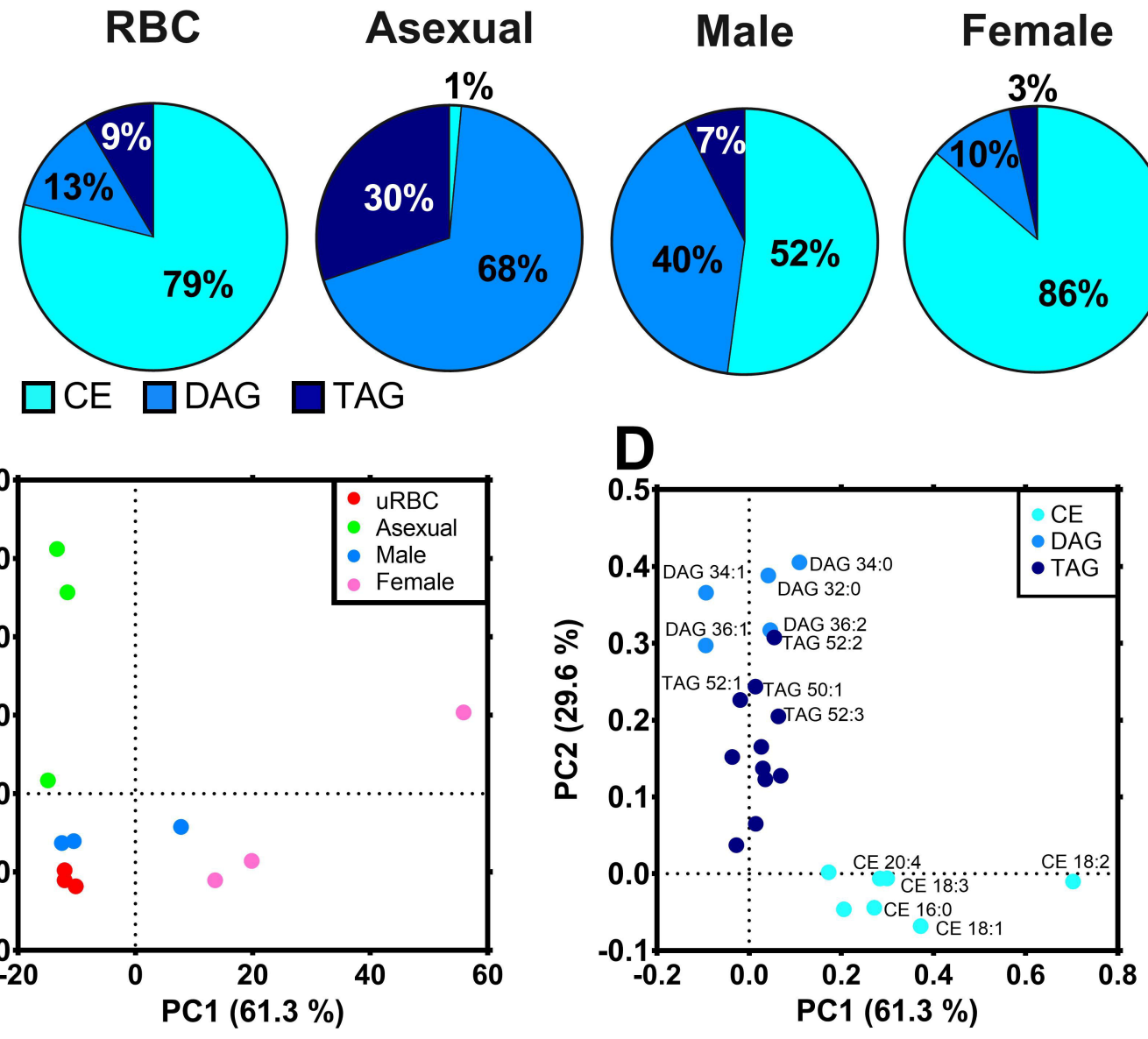


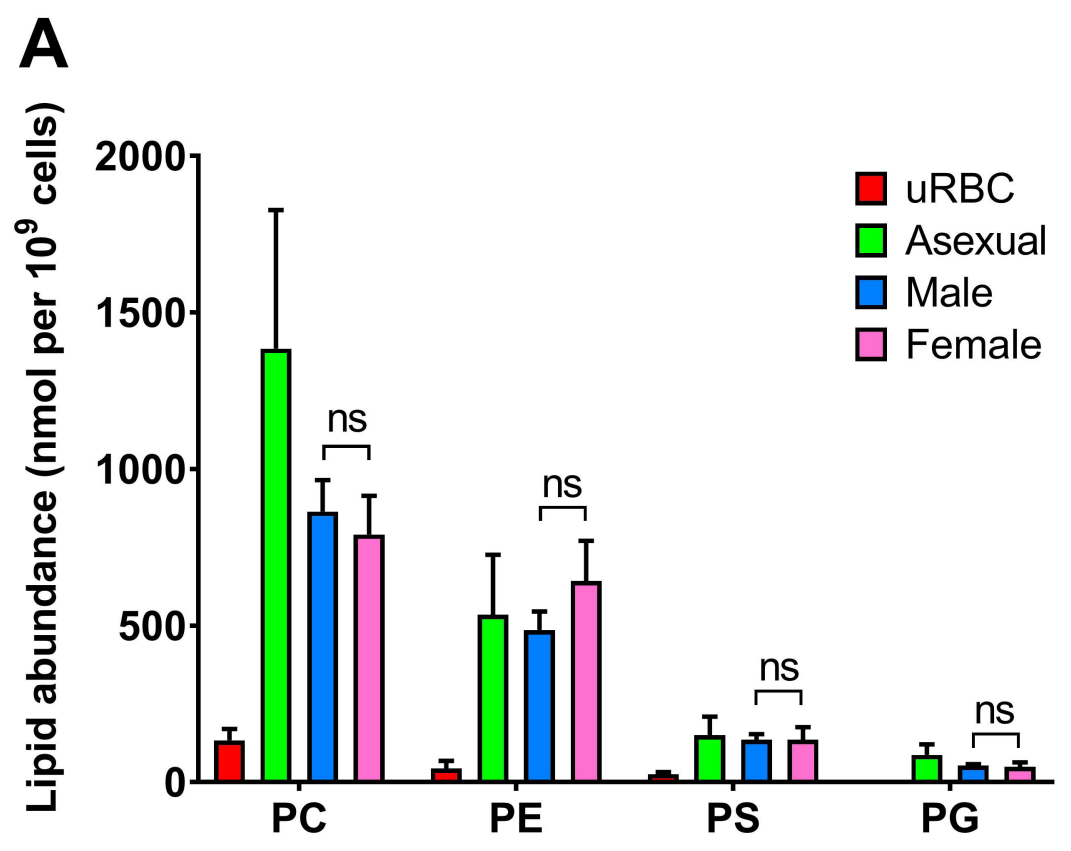

B

uRBC

Asexual

Male

Female
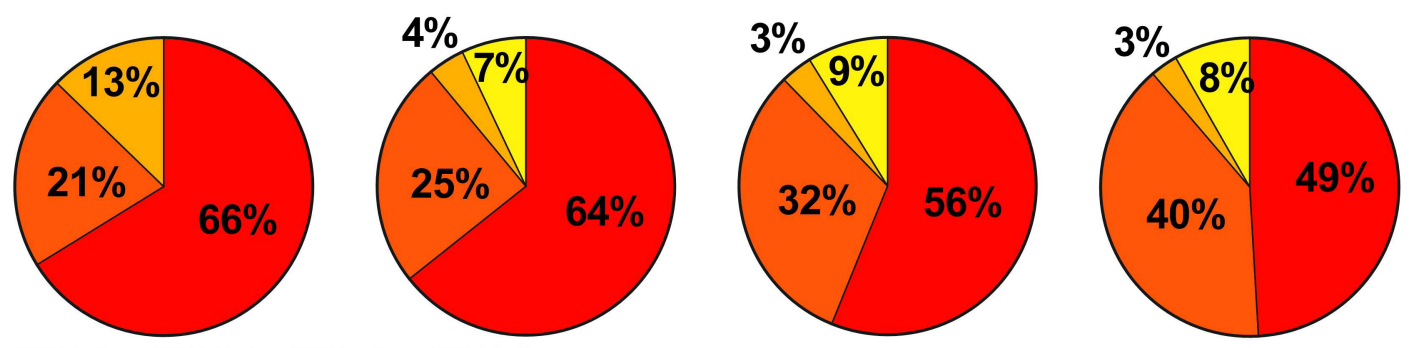

$\square \mathrm{PC} \square \mathrm{PE} \square \mathrm{PS} \square \mathrm{PG}$

C
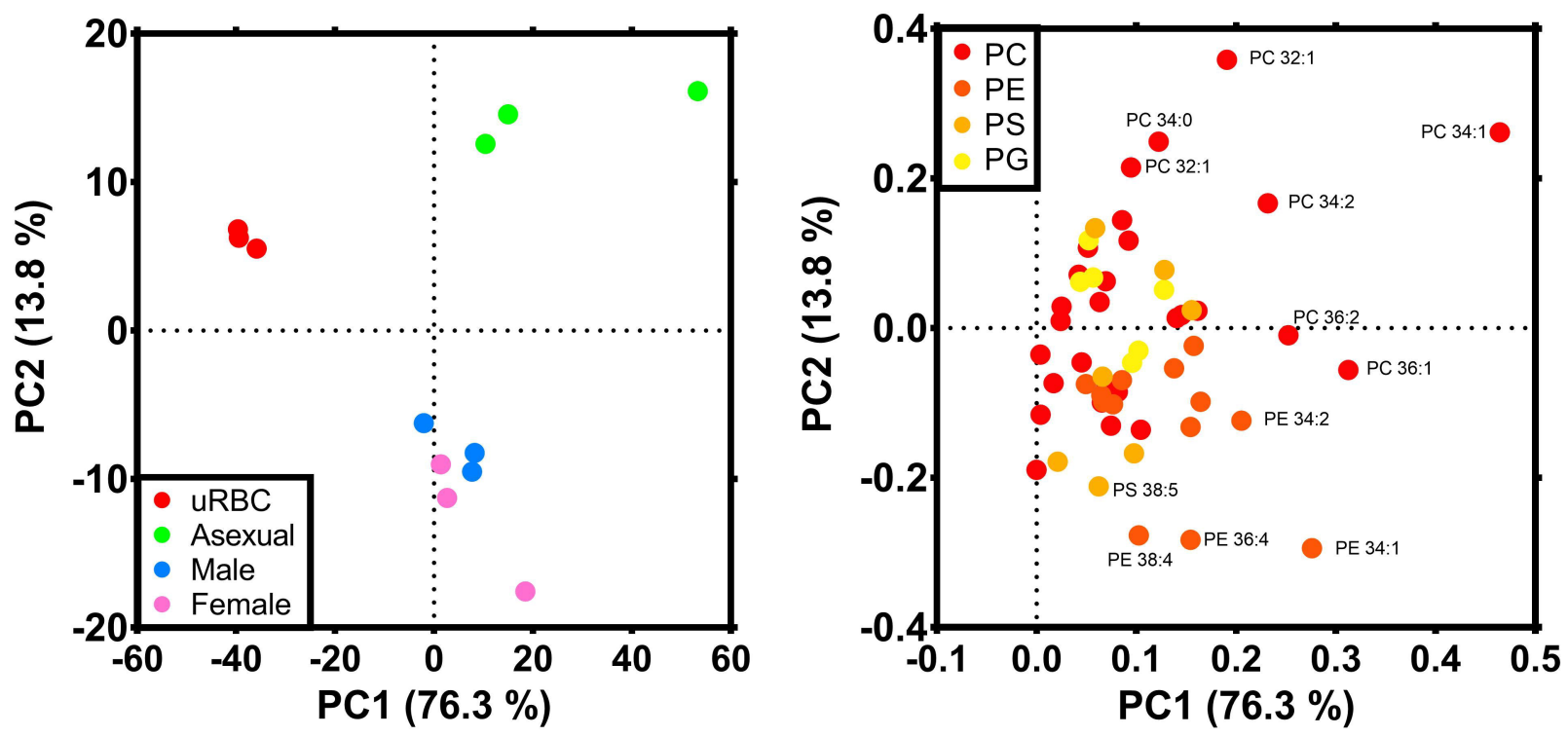
A

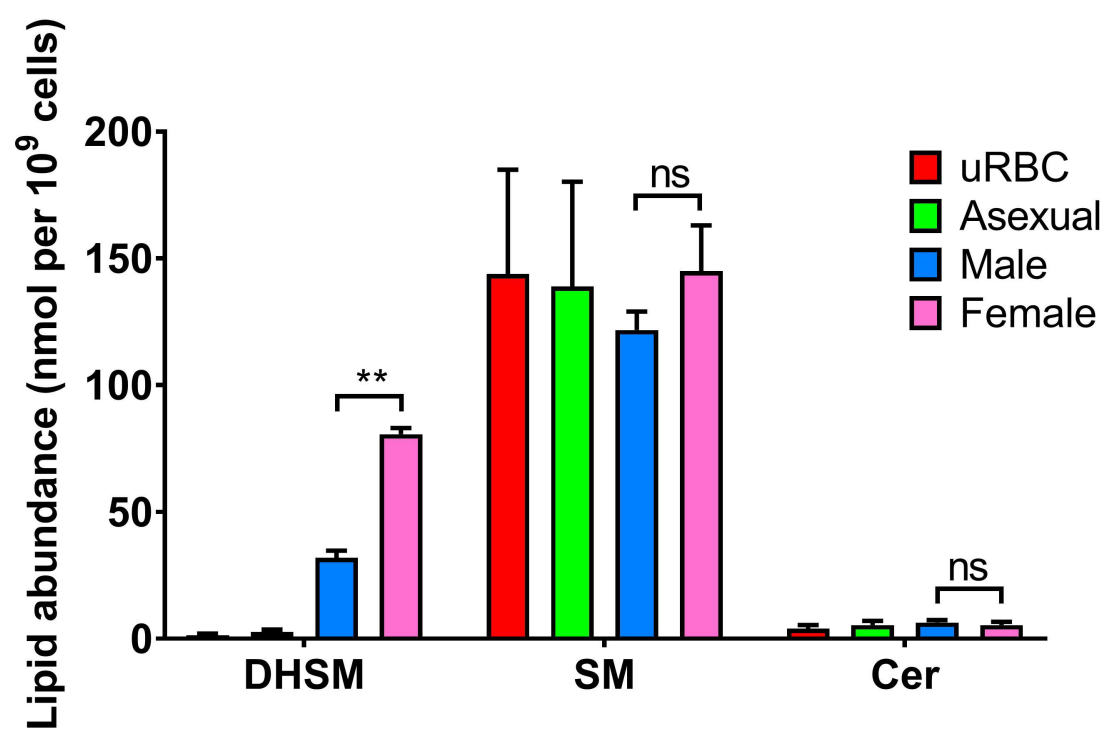

B
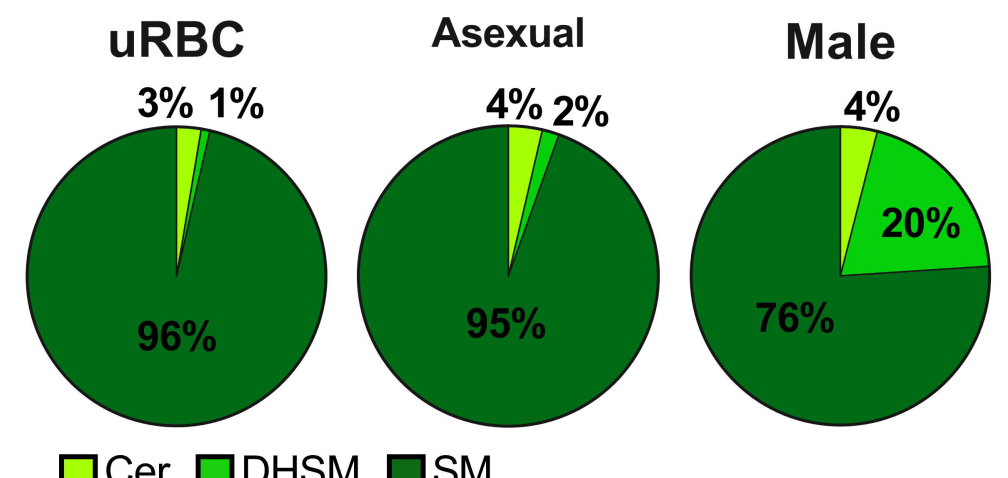

Female

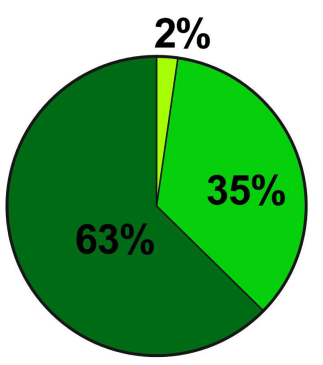

C
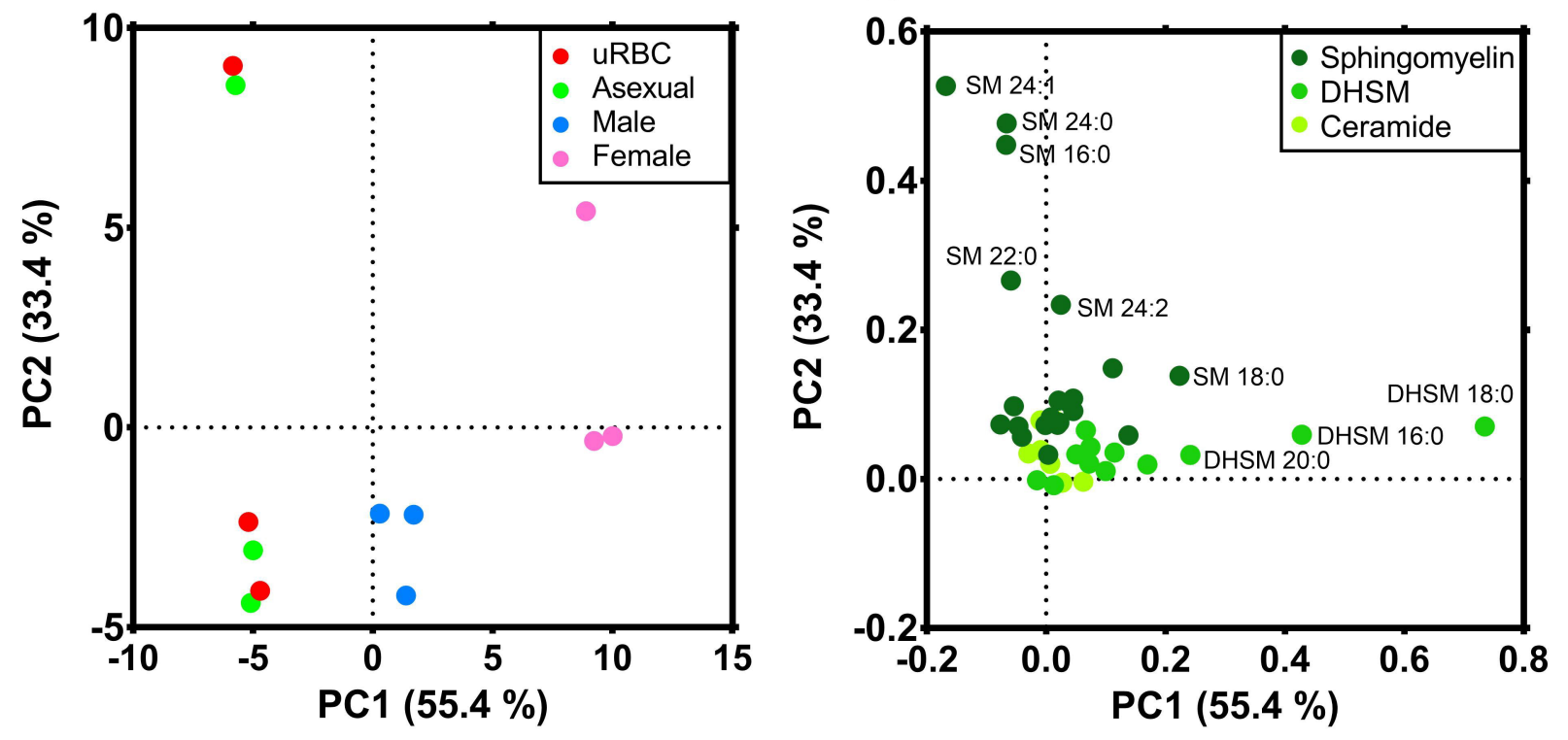
(which was not certified by peer review) is the author/funder. All rights reserved. No reuse allowed without pe

A

Male $>2 x$ Female Female $>2 x$ Male
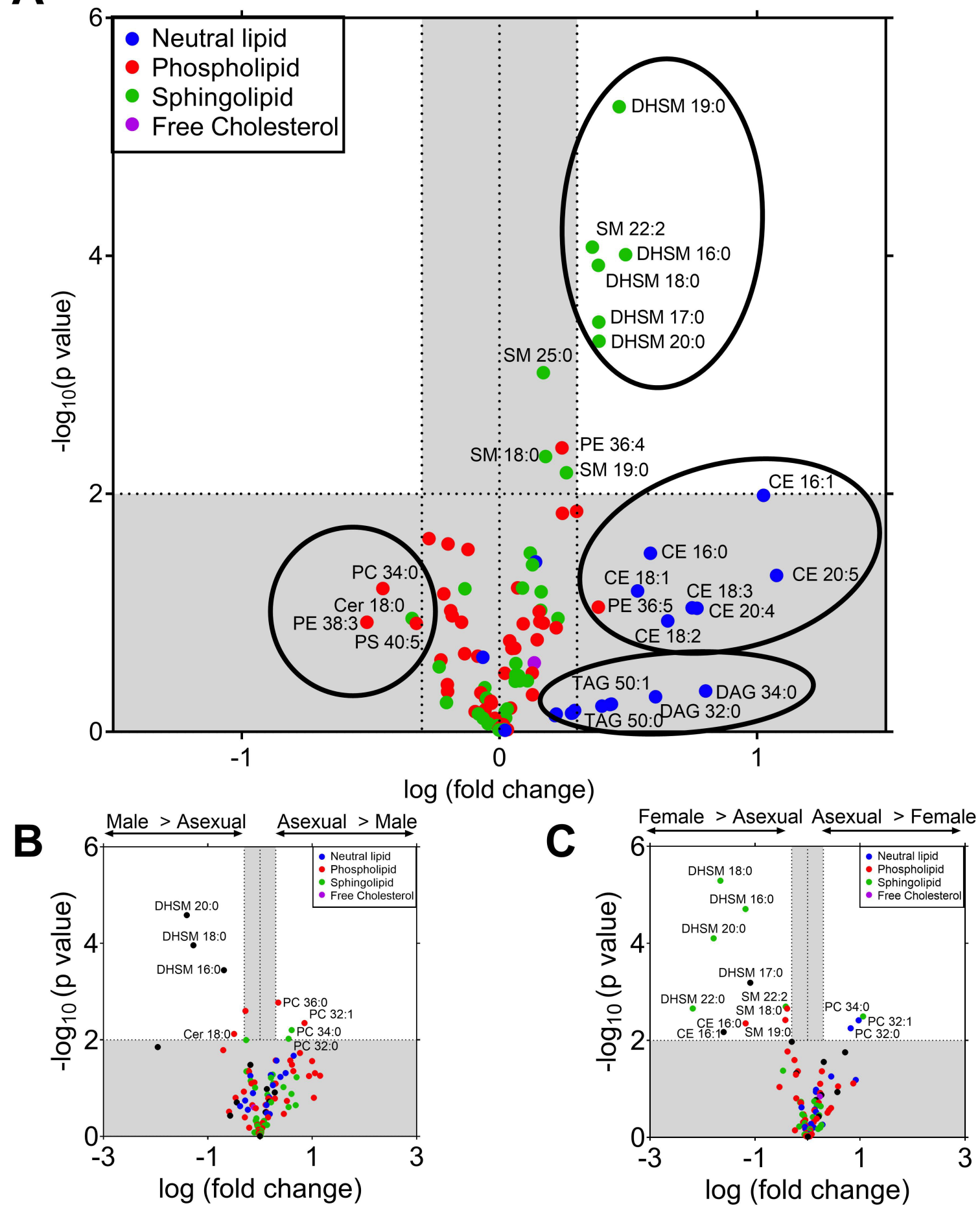

D
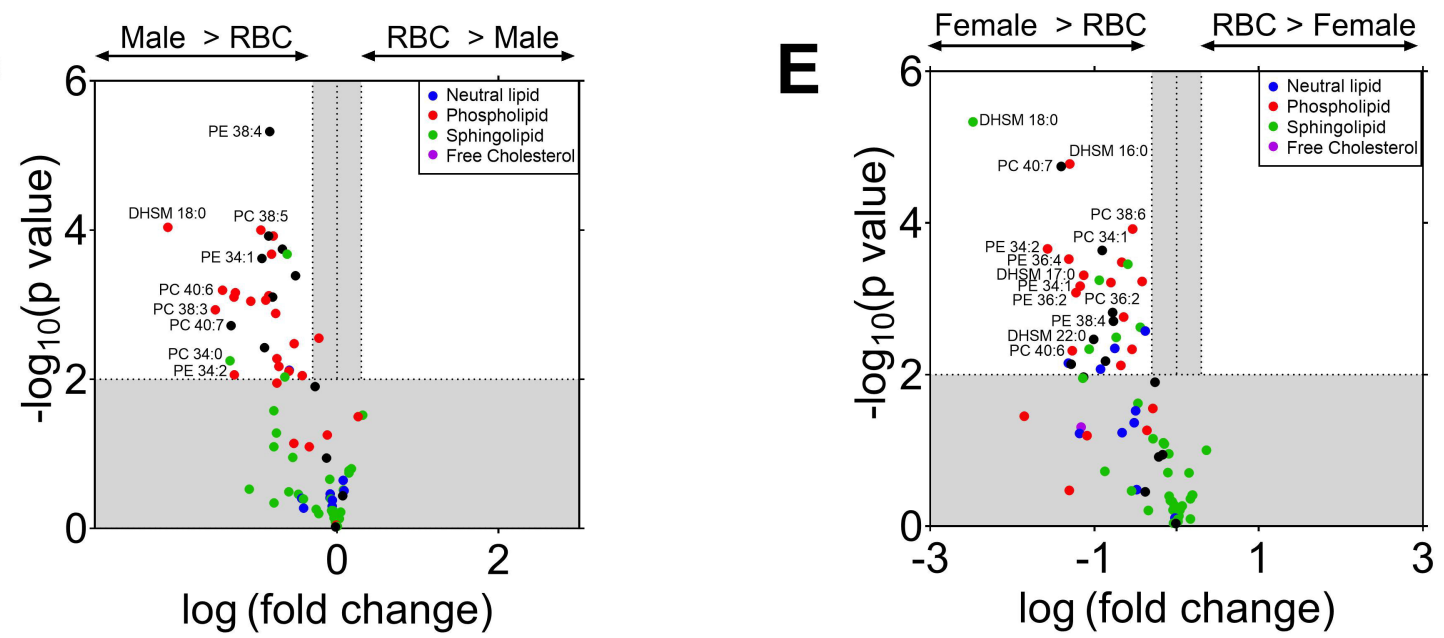
A

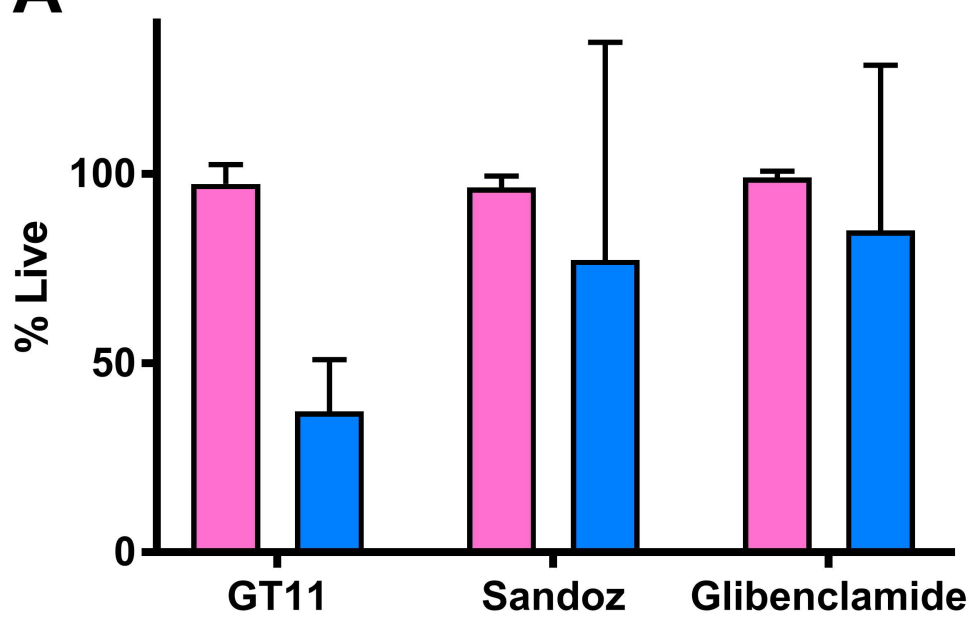

B

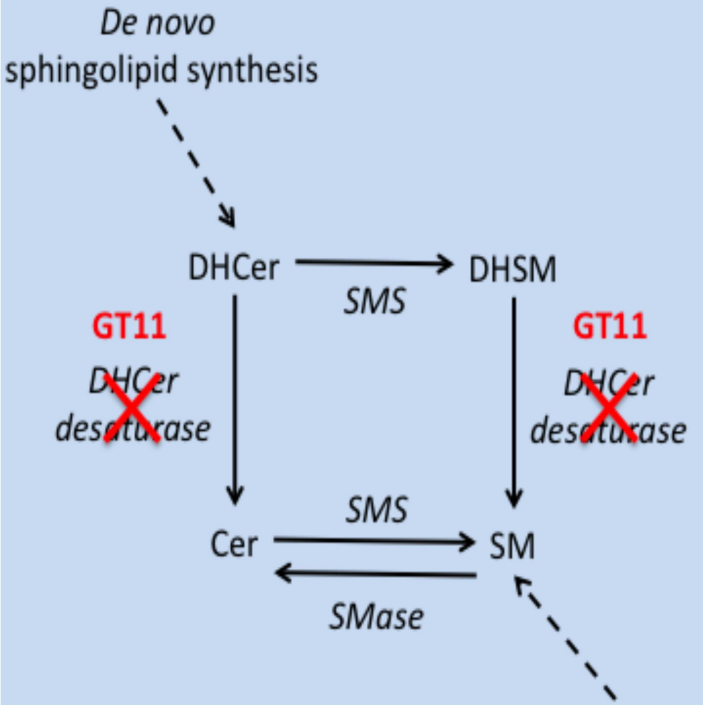

Host SM scavenging 


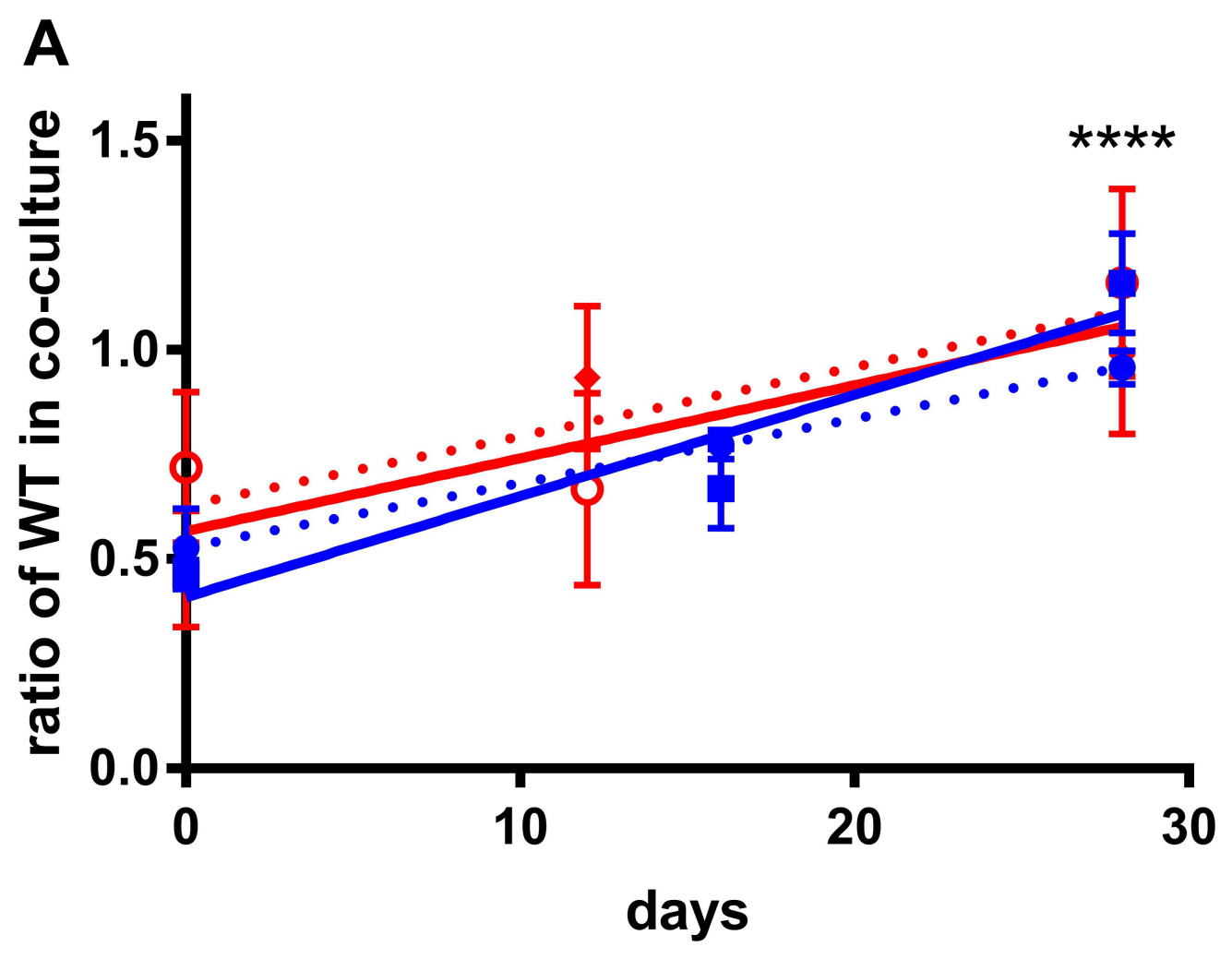

•. WT vs SMS1 KO clone E6 in ICM

$\rightarrow$ WT vs SMS1 KO clone E6 in CCM $\rightarrow$ WT vs SMS1 KO clone E10 in ICM

- •. WT vs SMS1 KO clone E10 in CCM
B

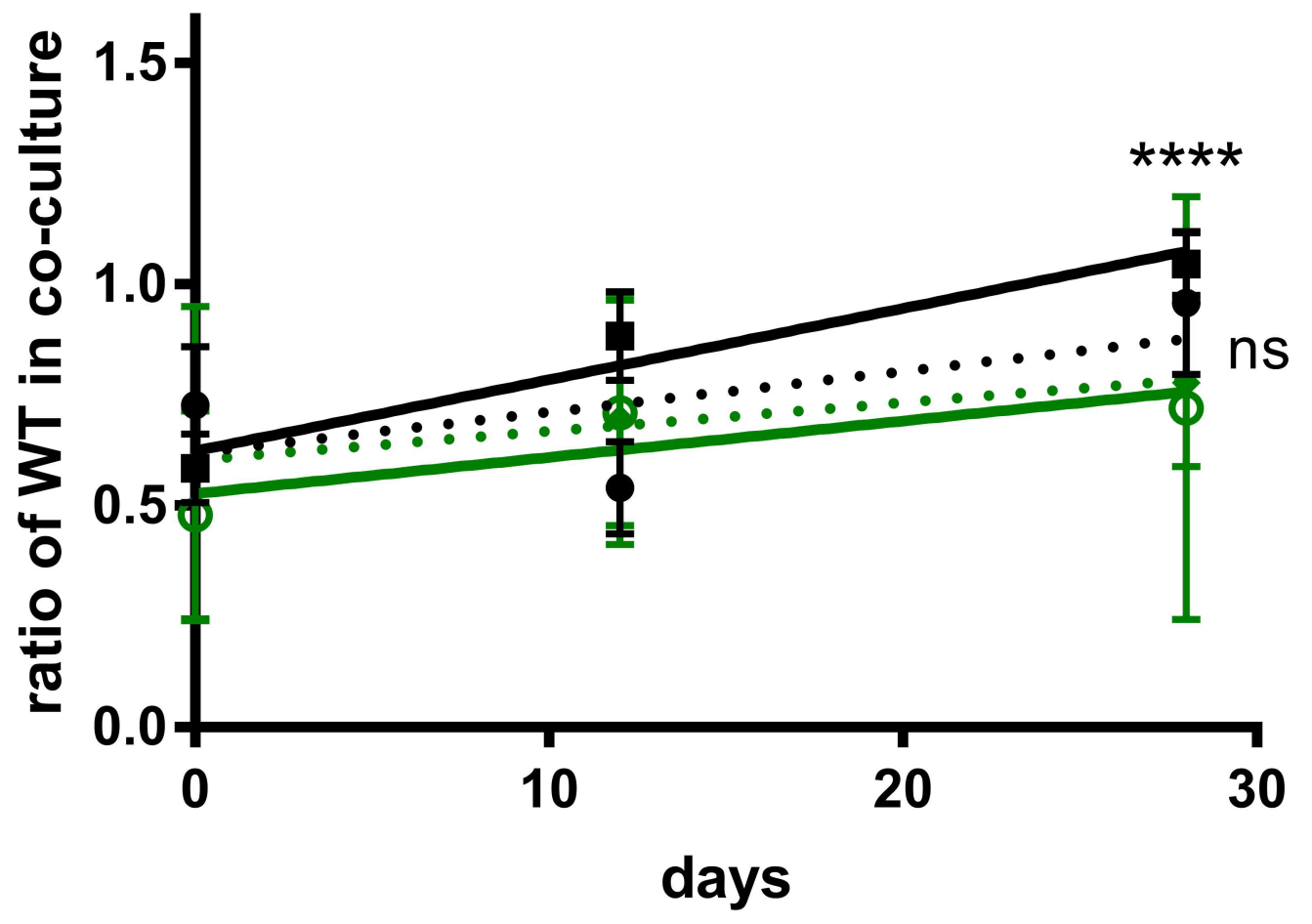

•. WT vs SMS2 KO clone D4 in ICM

$\rightarrow$ WT vs SMS2 KO clone D4 in CCM - WT vs SMS2 KO clone C9 in ICM

- WT vs SMS2 KO clone C9 in CCM 


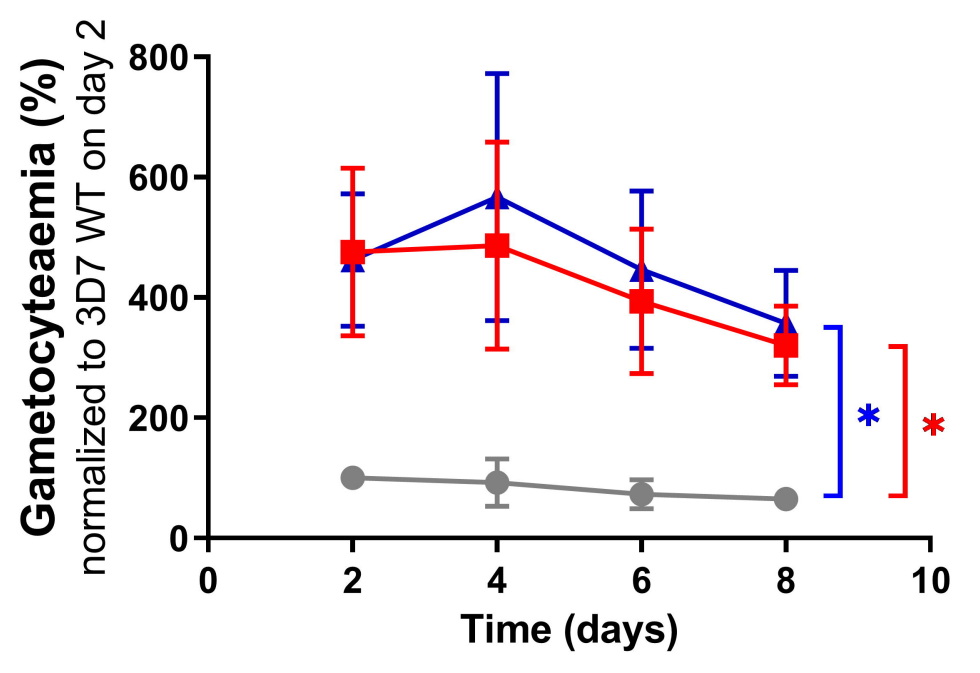

-c- 3D7 WT

- $3 \mathrm{D} 7 \mathrm{SMS} 1 \mathrm{KO}$, clone E6

+ 3D7 SMS1 KO, clone E10

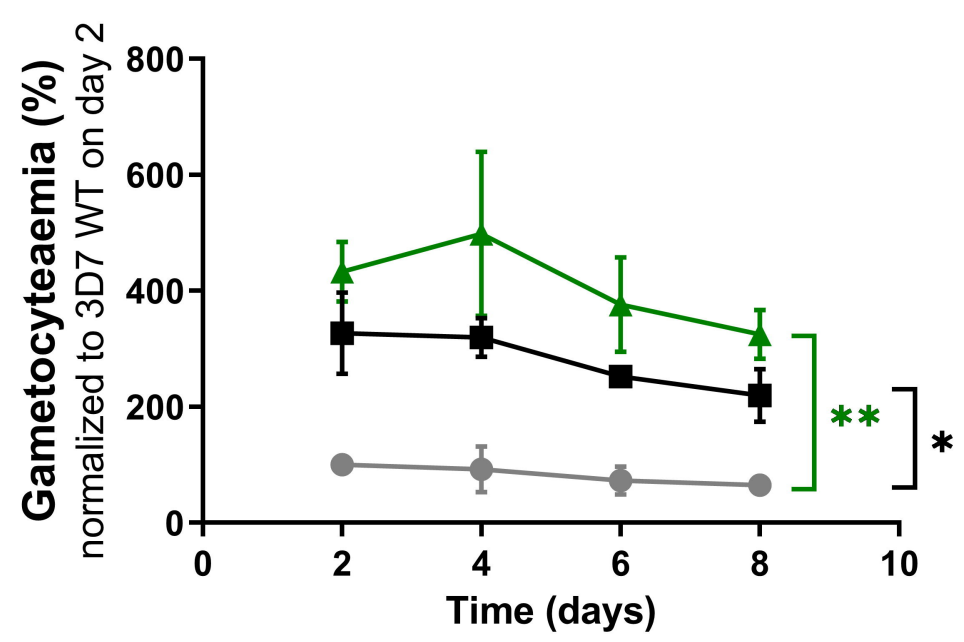

- $3 \mathrm{D} 7 \mathrm{WT}$

- 3D7 SMS2 KO, clone C9

\pm 3D7 SMS2 KO, clone D4

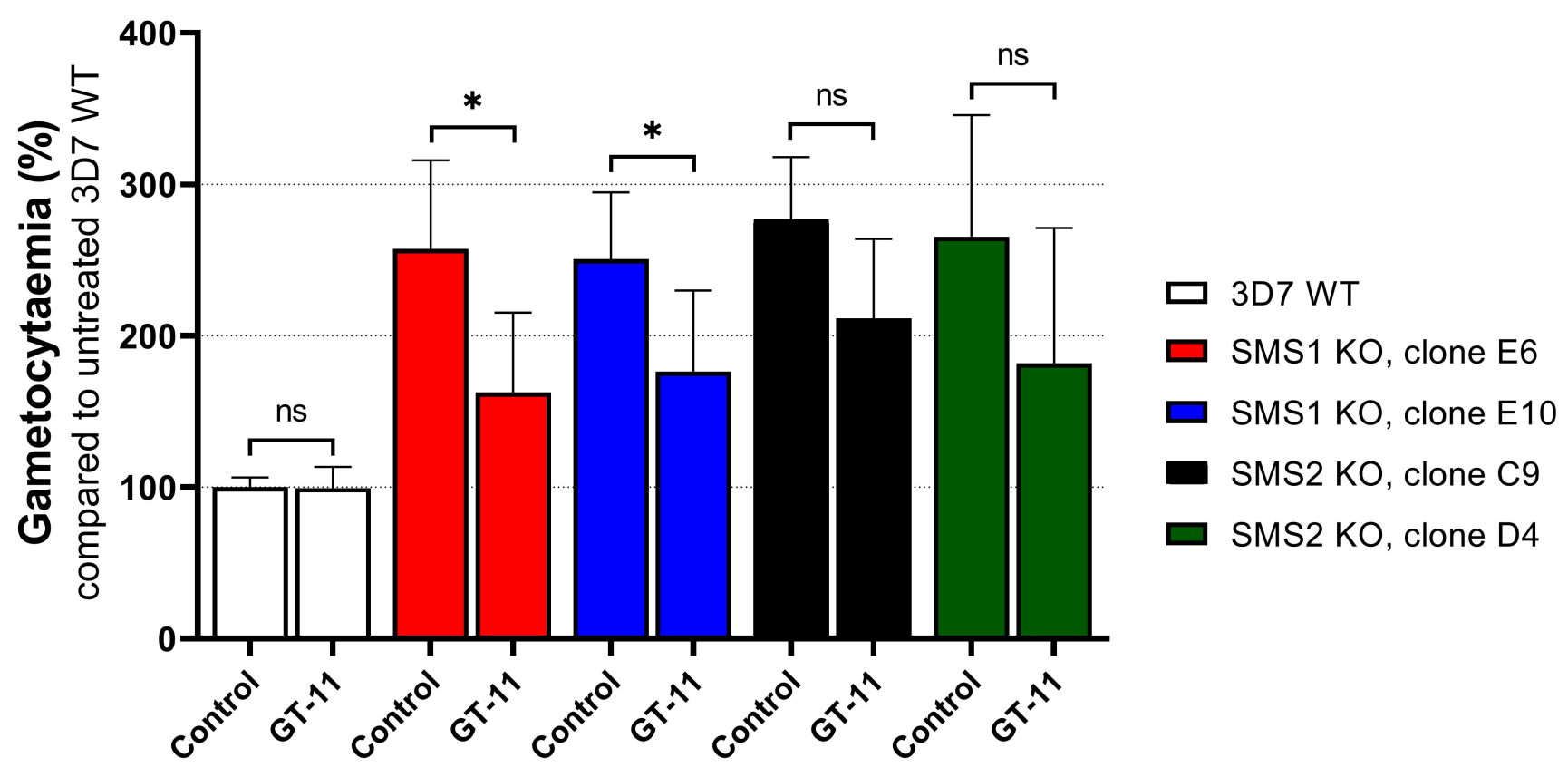



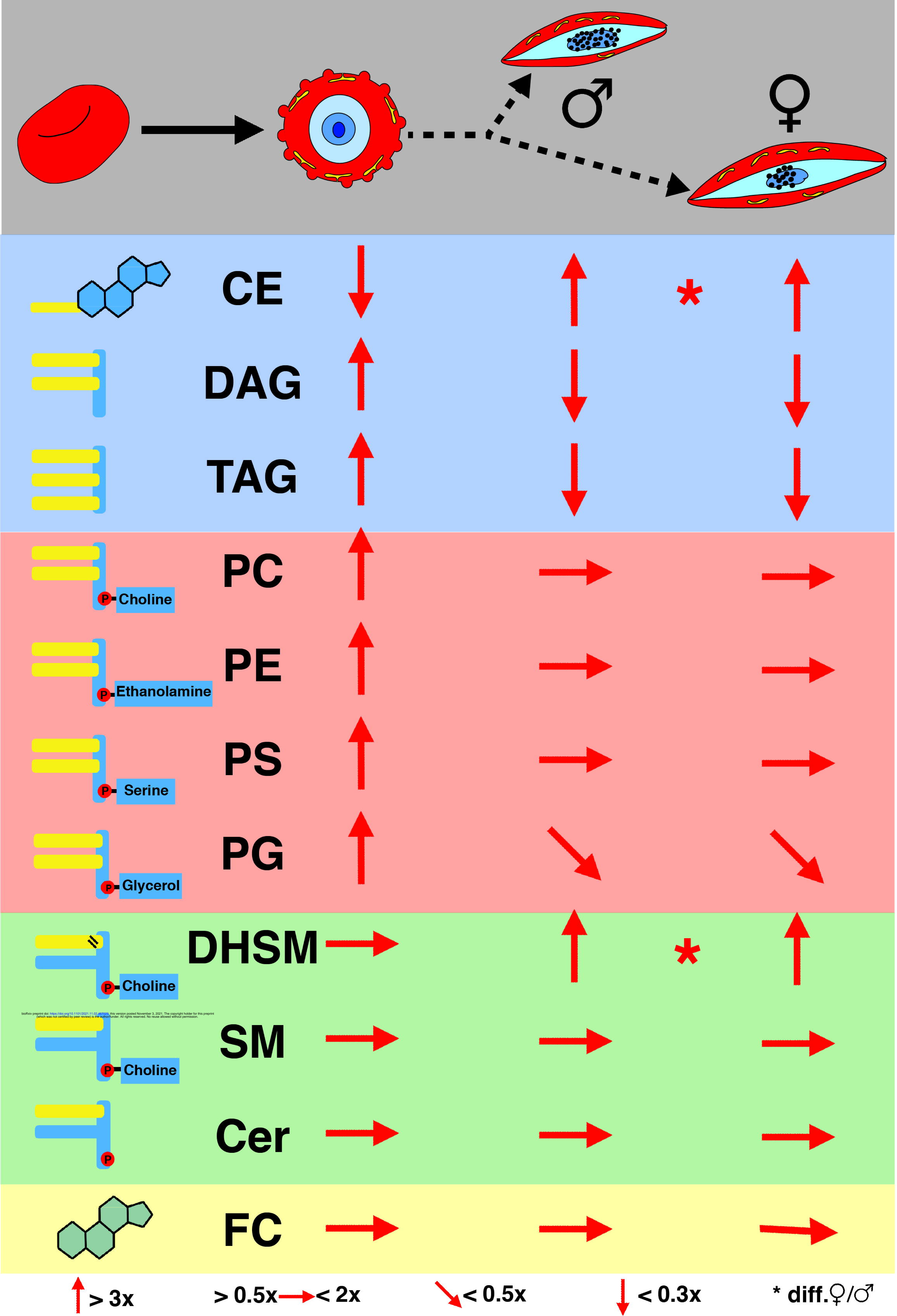